\title{
PBAR: A SUPERCONDUCTING MAGNET SPECTROMETER FOR COSMIC RAY ANTIPROTON STUDIES
}

\author{
A.D. TOMASCH ${ }^{\text {1) }}$, S.P. AHLEN ${ }^{2)}$, S.W. BARWICK ${ }^{3)}$, J.J. BEATTY ${ }^{2)}$, C.R. BOWER ${ }^{4)}$, \\ G. GERBIER (), R.M. HEINZ ${ }^{4)}$, D.M. LOWDER ${ }^{3)}$, S. MCKEE ${ }^{\text {) }}$, J.L. MILLER ${ }^{\text {() }}$, S.L. MUFSON ${ }^{\text {s), }}$ \\ J.A. MUSSER ${ }^{1)}$, P.B. PRICE ${ }^{3)}$, M.H. SALAMON ${ }^{6)}$, G. TARLE i) and B. ZHOU 2) \\ "Randall Laboratory, Department of Physics, University of Michigan, Ann Arbor, MI 48109-1120, USA \\ ${ }^{2)}$ Department of Physics, Boston University, 590 Commonwealth Avenue, Boston, MA 02215, USA \\ 3) Department of Physics, University of California, Berkeley, CA 94720, USA \\ 4) Department of Physics, Indiana University, Bloomington, IN 47405, USA \\ 5) Department of Astronomy, Indiana University, Bloomington, IN 47405, USA \\ 6) Department of Physics, University of Utah, Salt Lake City, UT 84112, USA \\ ") CEN Saclay, Gif sur Yuette, France
}

Received 3 April 1990

We describe the PBAR balloon-borne magnet spectrometer flown on August 13-14, 1987 to measure the abundance of cosmic ray antiprotons in the energy interval 100-1580 MeV at the top of the atmosphere. The limits first reported [S.P. Ahlen et al., Phys. Rev. Lett. 61 (1988) 145] have been improved [M.H. Salamon et al., Astrophys. J. 349 (1990) 78] to an overall limit of $\bar{p} / \mathrm{p}<2.0 \times 10^{-5}$ $(85 \% \mathrm{CL})$. We summarize the overall design and performance of the PBAR spectrometer, which had the unique ability to establish the mass of each singly charged cosmic ray, as well as to reject spurious antimatter candidates caused by hard scatterings within the instrument.

\section{Introduction}

The PBAR balloon-borne magnet spectrometer was built and flown to investigate the spectrum of cosmic ray antiprotons at energies below $2 \mathrm{GeV}$. Previous reports of an intensity of antiprotons at low energies several orders of magnitude greater than that expected from secondary production [1] had caused considerable interest, since such an excess would demand a new antiproton production mechanism or a drastic revision of cosmic ray propagation models. One particularly intriguing production mechanism proposed was the annihilation of supersymmetric particles such as photinos [2-4] or Higgsinos [4,5] within a massive relic particle halo surrounding the Galaxy. Since previous measurements were unable to identify particle species, we undertook the construction of PBAR to employ the rigidity-time-of-flight technique to measure particle masses at low energies, and extended the dynamic range of the instrument by using the rigidity-Cherenkov technique to measure mass at higher energies. The results of the measurements made during the balloon flight of August 13-14, 1987 have been reported [6,7], with no antiproton candidates observed. This results in an overall limit of $\bar{p} / p<2.0 \times 10^{-5}(85 \% \mathrm{CL})$ for the energy interval $100-1580 \mathrm{MeV}$ at the top of the atmosphere. In this article we summarize the design and performancs of PBAR and illustrate how it has been optimized for a high sersilivity, low background antiproton measurement.

\section{Theory of operation}

Fig. 1 illustrates the PBAR instrument. Scintillation counters $\mathrm{S} 1$ and $\mathrm{S} 2$ measure particle charge (via pulst height analysis of the photomultiplier tube signals) and

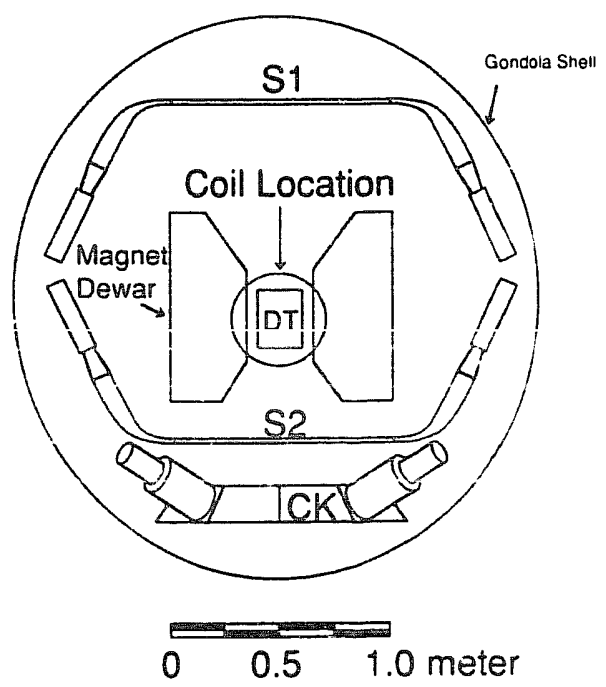

Fig. 1. Schematic of PBAR magnet spectroneter. 
time of flight (TOF). The particle's speed $\beta$ (in units of $c$, the speed of light) is then:

$\beta=\frac{L \sqrt{1+\tan ^{2} \theta}}{c \text { TOF }}$,

where $\theta$ denotes the angle between the particle trajectory and the zenith, and $L$ the distance between the two scintillators. For PBAR $L=150 \mathrm{~cm}$. The expression $\sec \theta=\left(1+\tan ^{2} \theta\right)^{1 / 2}$ has been used since $\tan \theta$ is easy to interpret when placing angle-dependent cuts on the data.

The drift tube array (DT) resides in the room-temperature bore of a high-field split-coil superconducting magnet, shown in cross section. Together they comprise the rigidity $(R)$ analysis system. For a particle with momentum $p$ and charge $z e$ :

$R \equiv \frac{p c}{z e}=\frac{\gamma m c^{2} \beta}{z e}$,

where $m$ denotes particle rest mass and $\gamma=\left(1-\beta^{2}\right)^{-1 / 2}$ is the Lorentz factor for a particle of speed $\beta$. For a magnetic field $B$ the trajectory of a particle with momentum $p$ has instantaneous radius of curvature $a$ for which:

$R=\frac{p c}{z e}=a|B|$,

where $1 \mathrm{kGm}=30 \mathrm{MV}$. In a plane normal to a direction $\hat{\boldsymbol{n}}$, a particle traversing a region of magnetic field exhibits a projected deflection angle $\theta_{D_{p}}$ for which

$\theta_{\mathrm{D}_{\mathrm{p}}}=\int \frac{\mathrm{d} l_{\mathrm{p}}}{a_{\mathrm{p}}} \approx \frac{1}{R_{\mathrm{p}}} \int(B \cdot \hat{n}) \mathrm{d} l_{\mathrm{p}}$,

where $\mathrm{d} l$ is the differential path length along the trajectory and the subscript $p$ denotes the projection of a quantity into the plane. Note that $R_{\mathrm{p}}$ can be extracted from the integration because the magnetic force does no work on the particle. Negative charges deflect in the opposite sense of positive charges, thus distinguishing particles from antiparticles. For PBAR the split-coil magnet design produced a magnetic field that was approximately uniform and constant in direction, with accepted particles moving normal to the field, so that $B \cdot \hat{B} \approx|B|$ and the subscripts denoting projection can be dropped. The quantity || $\boldsymbol{B} \mid \mathrm{d} l$ is commonly referred to as the "field integral" of the magnet, and was typically $\sim 2(\mathrm{kGm}) \sim 60 \mathrm{MV}$ for the PBAR flight. For small $\theta_{\mathrm{D}}$ the sagitta $(S)$ for the trajectory (the maximum displacement of an arc from its chord as shown in fig. 2) is approximately:

$S \approx \frac{l}{8} \theta_{\mathrm{D}} \approx \frac{l}{8 R} \int|B| \mathrm{d} l$.

For the PBAR spectrometer $l \sim 20 \mathrm{~cm}$. The sagitta error is related directly to the rigidity error: $|\delta R / R|=$ $|\delta S / S|$. A simple method for estimating the sagitta error due to tracking system errors is illustrated in fig.

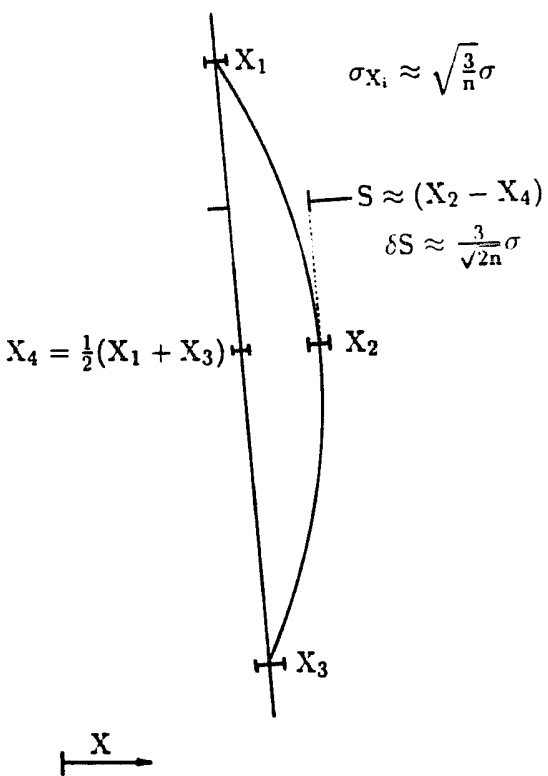

Fig. 2. A simple method for estimating the tracking error contribution to the error in sagitta.

2. Assume that $n$ measurements along the trajectory each with uncertainty $\sigma$ have been used to determine the points $X_{1}, X_{2}$, and $X_{3}$ each to an accuracy $\sigma_{X_{1}}$ $\sim \sqrt{2 / n} \sigma$. If the sagitta is defined as $\left(X_{2}-X_{4}\right)$ where $X_{4}=\frac{1}{2}\left(X_{2}+X_{3}\right)$, then the error in the sagitta is approximately $\delta S \sim(3 / \sqrt{2 n}) \sigma$. This simple approximation produces estimates in reasonable agreement with more detailed analysis [8]. A large number of high resolution position measurements therefore reduces the tracking system's contribution to the total rigidity error. Note that the fluctuations in $S$ are normally distributed, not the fluctuations in $R$.

In addition to providing TOF information, the scintillators also perform the important task of charge identification. The phototube pulse heights serve to distinguish different charge species, since energy loss scales as $(z / \beta)^{2}$. Consequently, higher $z$ species deposit more energy and yield more light at a given velocity than for $z=1$. By placing cuts on the scintillator pulse heights, $|z|>1$ particles can be excluded from the $\overline{\mathrm{p}}$ search analysis, or selected for study as desired.

Once $z, \beta$, and $R$ have been determined the particle mass $m$ is then calculated as:

$m=\frac{z e R}{c^{2}} \sqrt{\frac{1}{\beta^{2}}-1}$.

Thus, $R$, TOF, and $z$ completely characterize a particle traversing PBAR, providing momentum, mass, charge, and sign of charge. The spectrometer mass resolution is readily estimated as:

$\left(\frac{\sigma_{m}}{m}\right)^{2}=\left(\frac{\sigma_{R}}{R}\right)^{2}+\gamma^{4}\left(\frac{\sigma_{\beta}}{\beta}\right)^{2}$ 
Above $\beta=0.73$, the Cherenkov threshold in water $\left(n^{-1}\right.$, where $n$ is the refractive index), the Cherenkov counter (CK) provides additional velocity information via the relation:

$\frac{\mathrm{d} N_{\mathrm{pe}}}{\mathrm{d} x}=K z^{2}\left(1-\frac{1}{\left(n^{2} \beta^{2}\right)}\right)$.

Here $\mathrm{d} N_{\mathrm{pe}} / \mathrm{d} x$ denotes the number of photoelectrons (pe) detected per unit thickness of radiator by the counter's photomultiplier tubes (PMTs) from the conversion of primary Cherenkov photons (ignoring photons produced by delta rays), and $K$ the overall counter figure of merit. The radiator chosen should have its threshold velocity near where timing errors no longer allow for accurate TOF measurements, since a Cherenkov counter is best able to resolve velocities near its threshold. Water is well suited for PBAR in this regard, as well as being inexpensive and relatively easy to work w th. To obtain $N_{\mathrm{pe}}(z, \beta)$, the total number of photoelectrons detected for an incident particle of charge $z$ and speed $\beta$, eq. (8) must be integrated along the particle path through the radiator taking into account the slowing due to energy loss. This integral is well approximated by [9]:

$N_{\mathrm{pe}}=K z^{2} t(\theta)\left(1-\frac{1}{n^{2}}\right)\left(1-\frac{1}{\left(n^{2}-1\right)\left(\beta_{\mathrm{i}} \gamma_{\mathrm{i}}\right)\left(\beta_{0} \gamma_{0}\right)}\right)$,

where $t(\theta)$ is the thickness of radiator traversed above the Cherenkov threshold and $\beta_{i} \gamma_{i}\left(\beta_{o} \gamma_{o}\right)$ refers to the initial (exit or threshold) values for these quantities. If slowing is not appreciable, eq. (8) can be multiplied by the thickness of the radiator $t(\theta)$ and used to estimate $\beta$.

\section{Experiment trigger and electronics}

The PBAR trigger, shown in fig. 3, was designed to accept any charged particle which traverses S1, S2, andd DT. For an event to be digitized, each of the top tivo and bottom two drift tube layers must have contained a

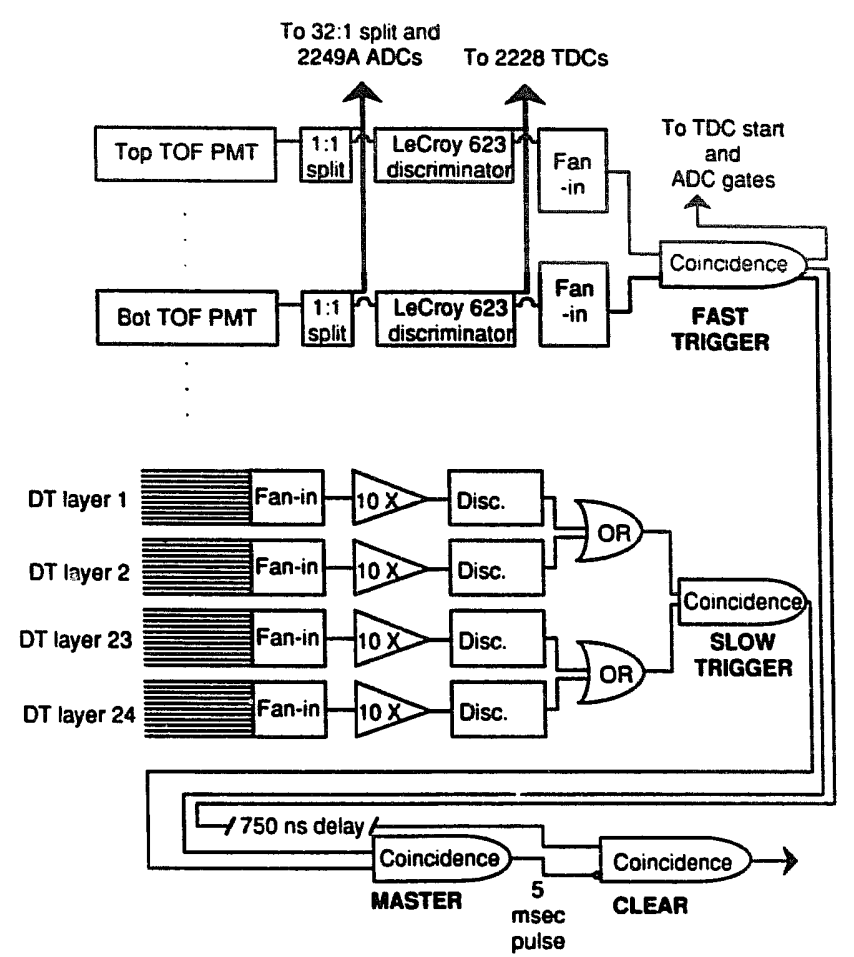

Fig. 3. Schematic diagram of the PBAR trigger logic.

hit tube in coincidence with at least one $\mathrm{Sl}$ phot ntube and at least one $\mathrm{S} 2$ phototube. To allow for the electron drift time within the drift tubes, a two-stage fast-slow scheme was used. A coincidence between S1 and S2 generated a "fast" trigger and a reset signal. In a drif tube coincidence was not received within $750 \mathrm{~ns}$, the reset signal cleared the trigger and acquisition electronics to await the next fast trigger. Receipt of a drift tube coincidence canceled the reset signal and permitted the event to be digitized. A valid trigger generated common start signals for the TOF and DT TDC systems, as well as gating the ADCs used to pulse height analyze S1, S2, and CK. With the exception of home-built passive signal splitters, fan-ins, and high voltage distribution boxes, commercial electronics were used throughout, with the majority comprised of CAMAC and NIM standard units. LeCroy 2249A ADCs and 2228 TDCs

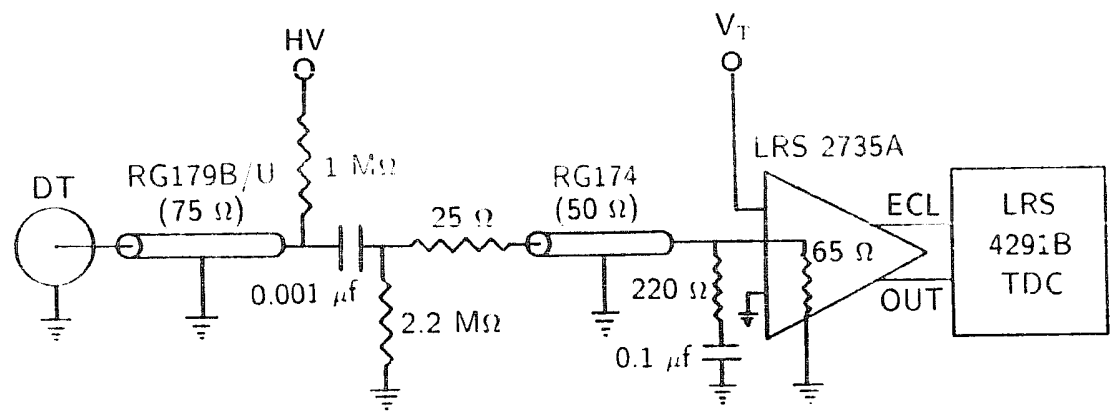

Fig. 4. Schematic diagram of the drift tube electronics. 
were used to digitize TOF pulse height and timing information, while a LeCroy 4290 TDC sjotem was used to digitize the drift tube timing information. A LeCroy $2249 \mathrm{~W} 11$ bit ADC was used to digitize the Cherenkov phototube signals.

A drift tube front end circuit is shown in detail in fig. 4. A total of 319 such channels were operational during the PBAR flight. The high voltage divider circuits were housed in custom-built metal boxes each containing 32 channels of divider circuitry. The drift tube high voltage was supplied via RG179 B/U coaxial cable connected to the drift tubes in the magnet bore. These also served as signal cables, with the high voltage decoupled from the signal network by a $0.001 \mu \mathrm{F}$ capacitor. This scheme eliminated the need to mount the divider and signal networks on the array, an impossible task due to the limited space within the magnet bore. The LeCroy 2735A amplifier/discriminator cards were housed in a home-built card crate and connected to the divider circuitry via RG174 coaxial cable. These cables all passed through a "switchboard" patch panel constructed with standard Lemo connectors. This arrangement allowed any drift tube to be connected to any 2735A channel and hence to any 4291B TDC shannel. This also permitted pulses to be observed directly from each drift tube before connecting it to its designated amplifier channel, allowing each tube to be checked individually for proper operation. The shielding provided by the use of coaxial cable throughout the system reduced interchannel cross-talk and pickup noise to a minimum. This allowed the threshold voltage for the discriminators (provided to the 2735A cards from an external power supply) to be set at a very low level without producing high singles rates. Typical singles rates at a flight threshold of $1 \mathrm{mV}$ at the amplifier front end were $3-5 \mathrm{~Hz}$ for one tube. A typical drift tube pulse, shown in fig. 5, had an amplitude of $\sim 70 \mathrm{mV}$ at this point in the circuit. The emitter coupled logic (ECL) differential pulses produce 1 by the $2735 \mathrm{~A}$ discriminator output were routed to the input of the 4291B TDCs in groups of 16 channels via twisted-pair ribbon cables. These also served to delay the drift tube signals $200 \mathrm{~ns}$ as required by the 4290 system when operated in common start timing mode. This entailed lying an additional $115 \mathrm{~kg}$ of ribbon cable, which could have been eliminated if the 4290 system had been operated in common stop mode, where the master trigger provides the stop signal for the drift tube timing. The decision to employ common start timing, even with the attendant weight penalty, arose from the fact that drift tubes operating in a high gain limited streamer [10] mode wili regenerate and produce a second pulse $-15 \%$ of the time as illustrated by the transient recording shown in fig. 6. In common stop mode this "after pulse" would cause spurious position information to be recorded since it is the time interval since the last pulse generated by

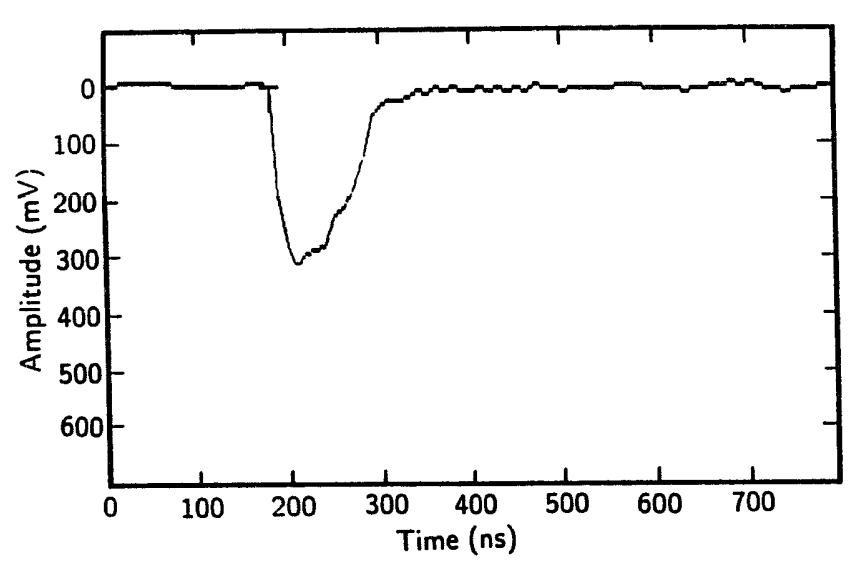

Fig. 5. Transient recording of a typical drift tube pulse. The peak signal level corresponds to $-70 \mathrm{mV}$ at the input of the LeCroy 2735A amplifier stage shown in fig. 4. The operating voltage was $2260 \mathrm{~V}$ (typical of flight operation) with a gas mixture of $\mathrm{Ar}: \mathrm{C}_{2} \mathrm{H}_{6} 1: 1$. The signal was produced by a cosmic ray muon, with the system triggered by a threefold scintillator coincidence.

the drift tube which is digitized. Common start timing is immune to this effect since it is the time interval from the start pulse until the first drift tube pulse received which is recorded. To save weight on future experiments, a pulse stretching circuit which allows the $\mathbf{4 2 9 0}$ system to be operated in common stop mode without sensitivity to after pulses has been developed. This system was used successfully for the drift tube readout of the SMILI [11] spectrometer flown in September of 1989.

Data acquisition and experiment control were performed in flight by an on-board DEC LSI-11/23 computer. The digitized data were sent to a custom-built pulse code modulation (PCM) encoder which buffered the incoming data and converted it to a serial PCM bit

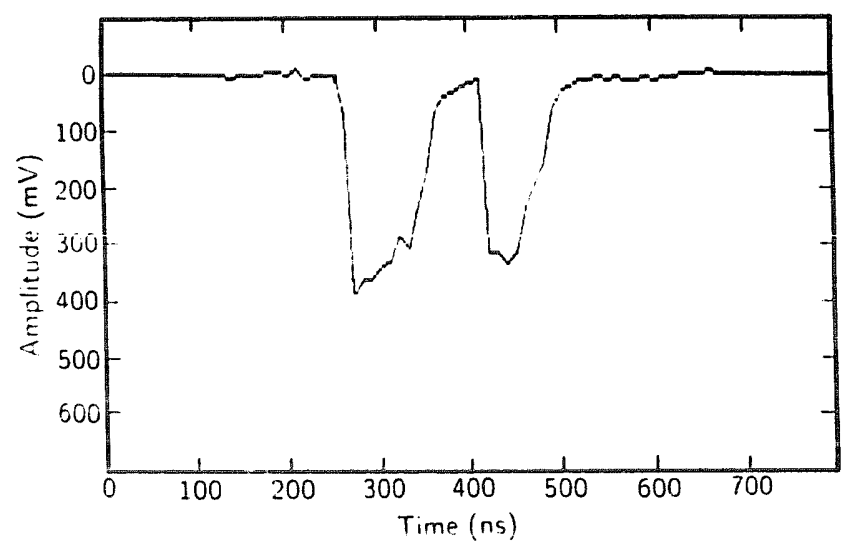

Fig. 6. Transient recording of a drift tube pulse for which a second regeneration pulse or "after pulse" is present. This occurs $-15 \%$ of the time. Voltage and gas mixture ar the same as in fig. 5 . 
stream which was then transmitted to a ground-based data acquisition station to be recorded on tape. Telemetry could be accomplished by radio transmission (flight mode) or via a single $50 \Omega$ coaxial cable (ground evaluation mode) at a rate of $32 \mathrm{kbps}$. The ground station consisted of a PCM synchronizer/demodulator and a DEC PDP-11/73 computer running a specially modified version of the Fermilab MULTI data acquisition system. During operation, the acquisition system made environmental and diagnostic information available in real time, including continuously updated histograms of scintillator and Cherenkov pulse heights and TDC timing distributions. It also monitored critical environmental and performance data and could sound an alarm in the system operator if such a "watched variable" went out of its preset nominal operating range during instrument operation.

\section{Time of flight scintillators}

The TOF scintillators $S 1$ and S2, shown in fig. 7, were of modular construction and separated by a baseline of $150 \mathrm{~cm}$. S1 consisted of three modules, while S2 contained two. Each module was composed of a fast Bicron BC420 plastic scintillator "slab" with active dimensions $100 \mathrm{~cm} \times 25 \mathrm{~cm} \times 2.54 \mathrm{~cm}$. A Philips XP2020 photomultiplier tube (PMT) viewed each end through a bent twisted triplet Lucite lightpipe. Each PMT detected $\sim 50$ photoelectrons for a relativistic muon. Both the scintillator and the PMTs used were chosen as the best known to date for timing applications. The BC420 scintillator exhibits a rise time of 0.5 $\mathrm{ns}$, a decay time of $1.5 \mathrm{~ns}$, and an attenuation length of $1.1 \mathrm{~m}$, permitting good light collection efficiency over

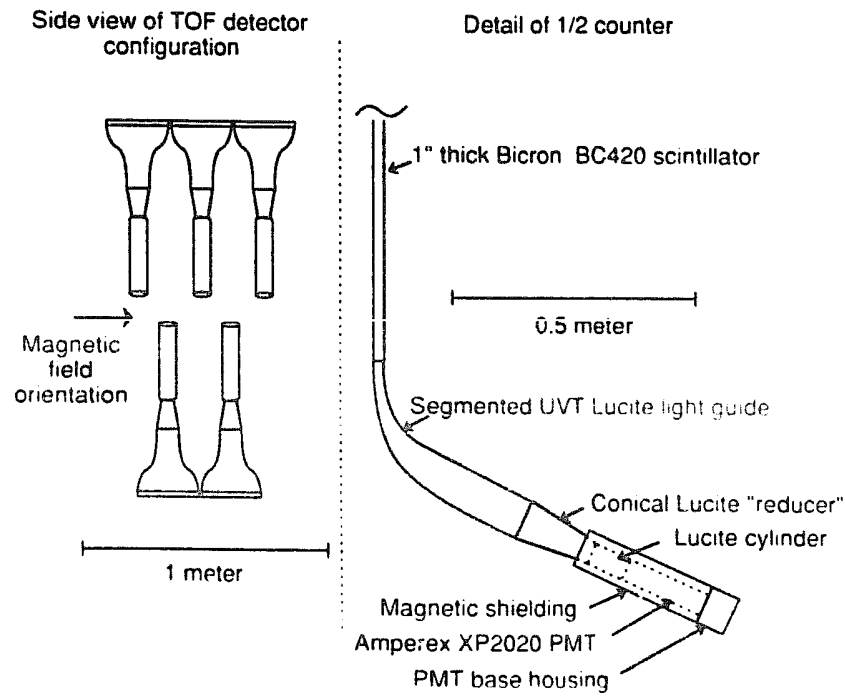

Fig. 7. Arrangement of the TOF system, and detail of a single counter.

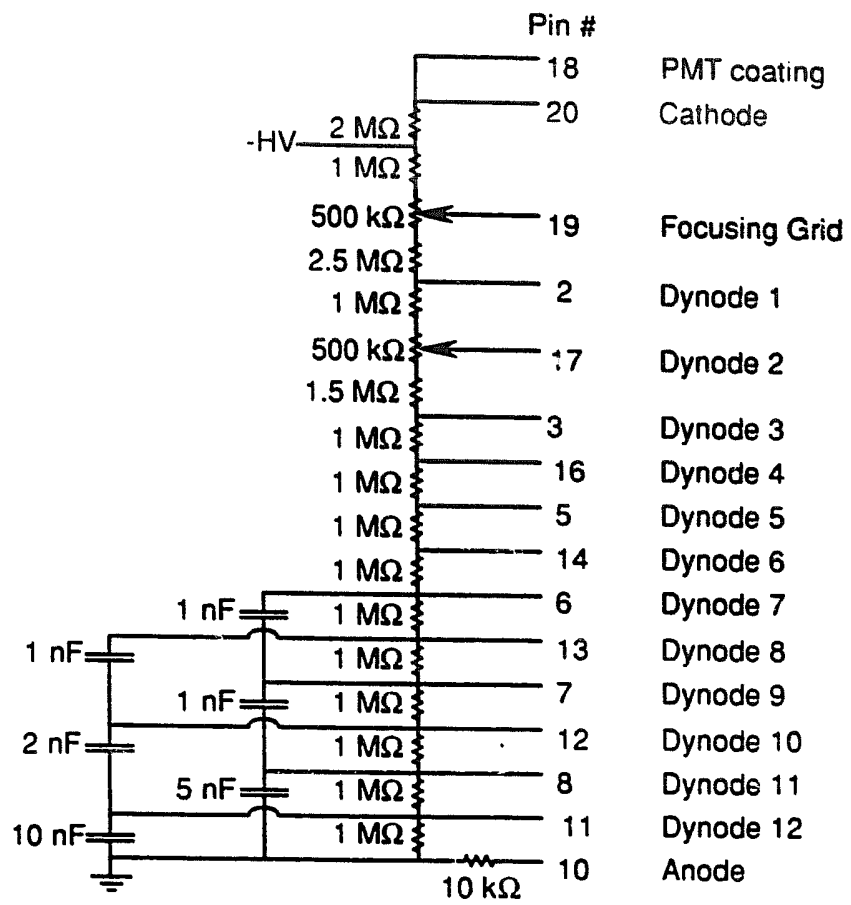

Fig. 8. Schematic diagram of the TOF photomultiplier high voltage divider and signal network.

large counters. The XP2020 provides both a very high gain $\left(10^{7}\right)$ and a very fast rise time (1.5 ns).

The phototube high voltage divider circuit is shown in fig. 8, and is based on a design that had given the best timing resolution in previously reported tests [12]. Each phototube-base combination was tested for gain characteristics and dark current. Based on these measurements, the voltages at the focusing electrodes were adjusted for optimal performance.

Once suitably fast scintillator and phototubes have been chosen, the geometry of the scintillator/light guide system must be optimized to ensure the best possible timing resolution. It is important to realize that maximum light collection efficiency is not necessarily compatible with optimal timing. For good timing, the detected photons should come as directly as possible (smallest number of internal reflections) from their point of origin, and ideally only these "direct" photuns should be detected. since slow photons obscure the time-pulse height correlations required to make accurate timing walk corrections. The PBAR TOF counters were comprised of long narrow slabs, thus ensuring that light emerging at the ends was moving nearly parallel to the counter and hence was dominated by direct photons. This geometry also ensured that the response was uniform across the counter, eliminating "hot spots" near the edges. The connection between the light guide and the PMT was made with two Lucite pieces as shown in fig. 7. The cone-shaped "reducer" produces a focusing whereby photons coming at large angles from the light 


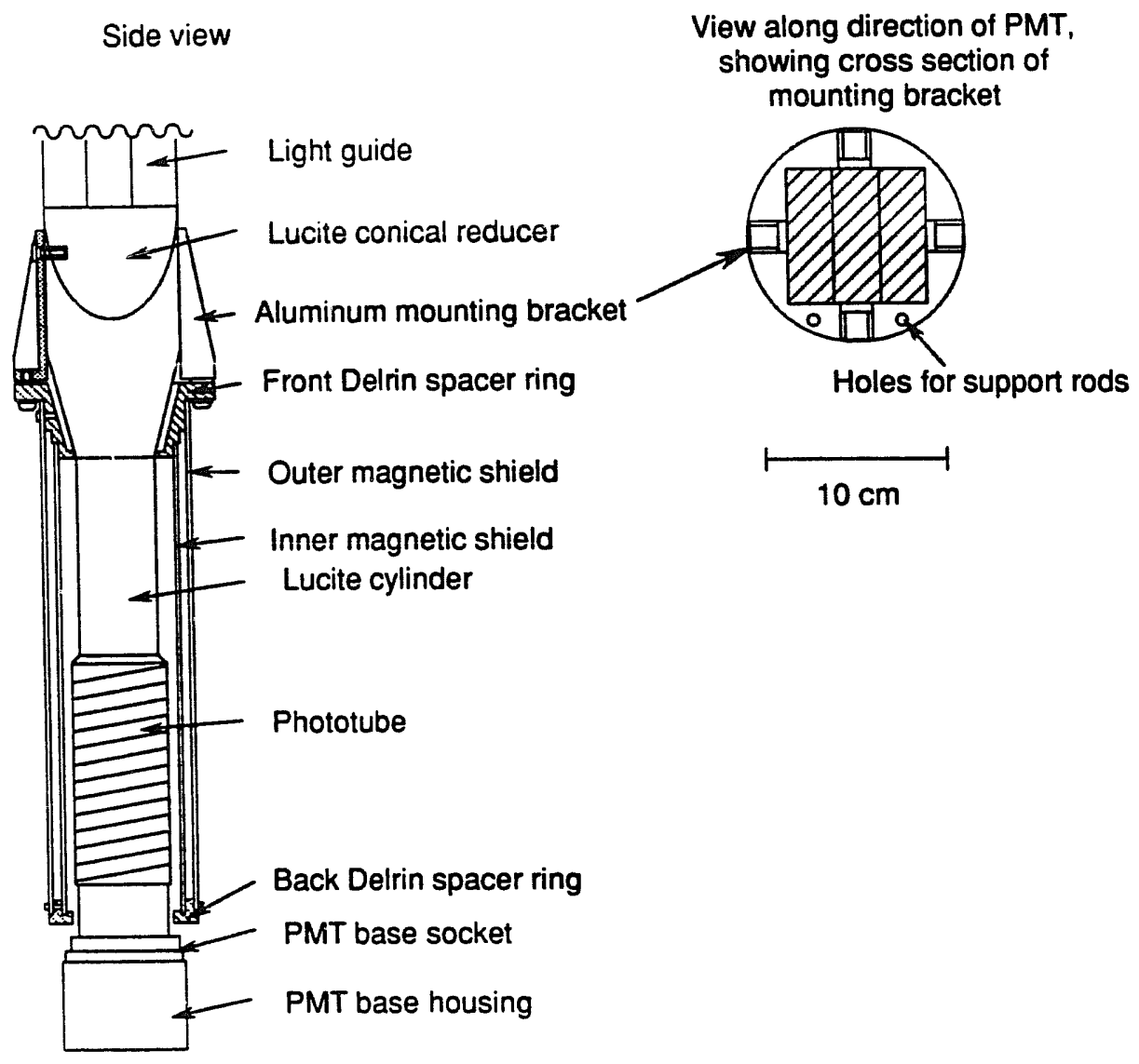

Fig. 9. Detail showing TOF magnetic shielding and associated mechanical attachment fittings.

guide are not internally reflected and escape, further enriching the overall prompt photon content of the detected light. This has been demonstrated [13] as necessary to achieve the best timing resolution for large counters. The cylindrical Lucite segment was required to bring the light into the magnetic shielding (fig. 9) which extends $10.2 \mathrm{~cm}$ beyond the end of the phototube window.

The segmented light guides, shown in fig. 10, were custom-made from strips of $2.54 \mathrm{~cm}$ thick Lucite 76.20 $\mathrm{cm} \times 8.25 \mathrm{~cm}$. All cut surfaces were thoroughly sanded and polished. The lightpipes were bent $65^{\circ}$ in order for the modules to fit within the gondola shell. The strips were shaped by heating to $150^{\circ} \mathrm{F}$ in an oven, and then bending and clamping them in a specially constructed jig. A set of three bent strips was then welded into a unit with ethylene dichloride solution, producing an optically transparent joint. The ends of these assembled light guides were machined to length and carefully polished. The same bending and machining fixtures were used for all of the guides, ensuring that all the guides were very nearly identical.

The completed TOF counters were built to withstand a vertical acceleration of $10 \mathrm{~g}$ and a horizontal acceleration of $5 \mathrm{~g}$ ( $\mathrm{g}$ is the acceleration of gravity) in order to meet the structural standards set by the $\mathrm{Na}$ - tional Scientific Balloon Facility (NSBF) for balloonlofted payloads. This required that they be assembled with a glue which provides not only a good optical coupling between scintillator and Lucite, but excellent

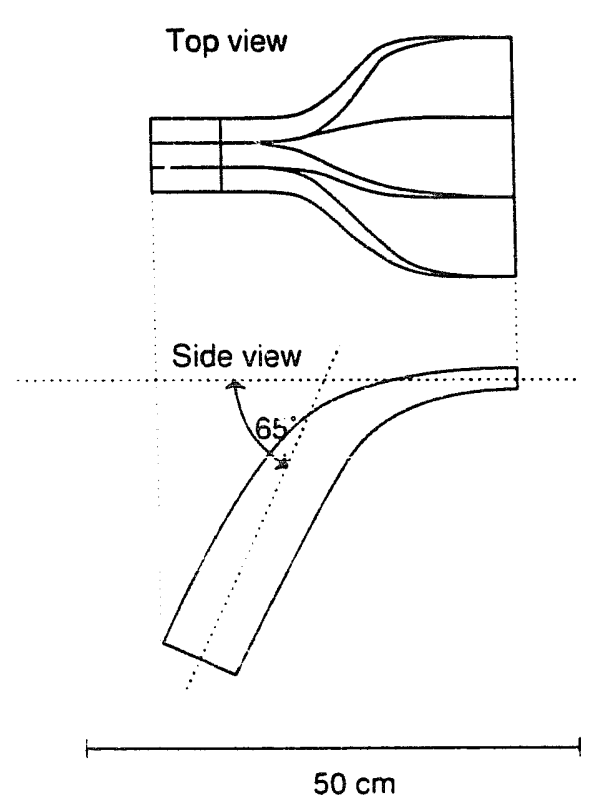

Fig. 10. Geometry of the segmented light guides used for the TOF scintillation counters 
mechanical strength and flexibility as well. Standard optical cements used for accelerator-based scintillation counters were tried and proved to be too brittle. Eventually Hartel HE17017 [14], a flexible urethane-based adhesive, was found to be ideal. Optically, HE17017 is a near perfect match to Lucite. While providing excellen strength, HE17017 can still be removed from a Lucite or scintillator surface with ethyl alcohol even when fully cured, permitting a broken joint to be repaired. One such scintillator-light guide joint was repaired in the field shortly before flight. The completed counters were wrapped in a black Tyvek-based paper [15]. Rather than wrap the counters in aluminum foil under the light proof layer, as is usually done to improve light collection, the black paper was directly in contact with the scintillator to absorb photons at large angles to the scintillator surface that might degrade the timing response. The cylindrical light guides within the magnetic shields were wrapped in aluminum foil to minimize light losses once the prompt photons had been selected out by the reducer cone. The counters then received a final overall wrap of Scotch Wrap black plumbing tape which provided excellent light exclusion, as well as additional mechanical strength.

The PMTs were surrounded by two magnetic shields as shown in fig. 9. This shielding permitted the tubes to be operated with no measured degradation of performance in the $\sim 200 \mathrm{G}$ fringe fields produced by the superconducting magnet. Because of the high ambient field, a two-stage shield with a low permeability $(\mu)$ soft iron outer shield surrounding a high $\mu$ inner shield was employed. The iron outer shield material is relatively immune to saturation, but provides insufficient attenuation of the field. The high $\mu$ inner shieid, which would saturate in the ambient field, provides the required attenuation once the outer shield has reduced the field to a level below the saturation field of the high $\mu$ material. In addition, the tubes themselves were wrapped with three layers of magnetic shielding foil. Finally, an innermost layer of lead foil was wrapped around each tube to protect it from contamination [16] by helium gas which could be present in the gondola atmosphere if the magnet cryogenic system were to develop a leak. Also shown in fig. 9 is the mechanical support arrangement used to attach the shielding to the PMT-light guide assembly. Since the tubes were operated at negative high voltage, the shields were electrically grounded and insulated from the phototube surface (maintained at negative high voltage) with teflon rings.

\section{Rigidity spectrometer system}

The rigidity spectrometer system consisted of a drift tube tracking hodoscope located in the uniform field region of a two-coil superconducting magnet [17]. The cryostat design, sketched in fig. 11, allows particles to pass through the uniform field region betwee'! the coils without penetrating dewar material. Also shown in fig. 11 is the cryogenic boiloff plumbing. A block diagram

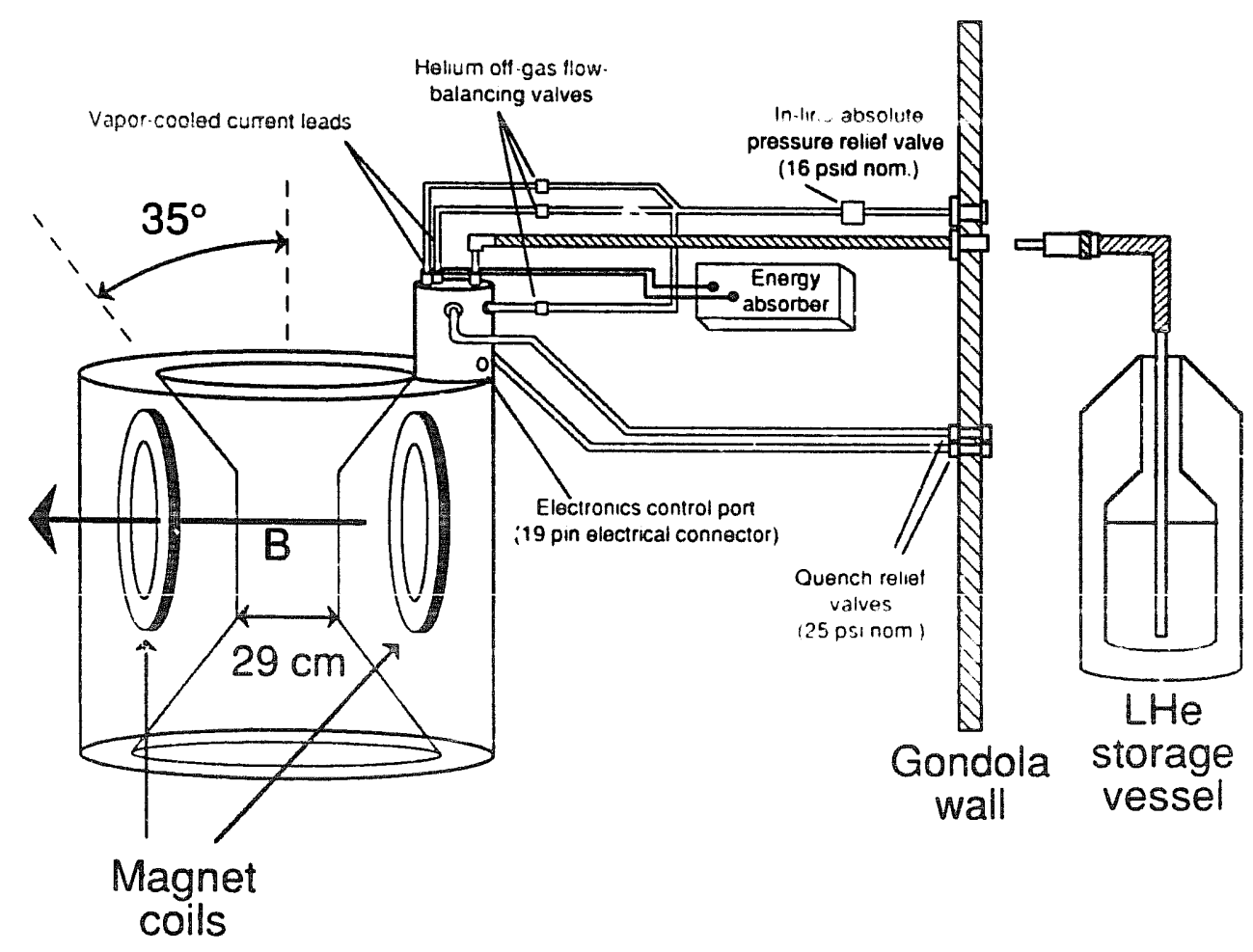

Fig. 11. Schematic overview of the superconducting magnet, dewar. and cryogenic systen. 


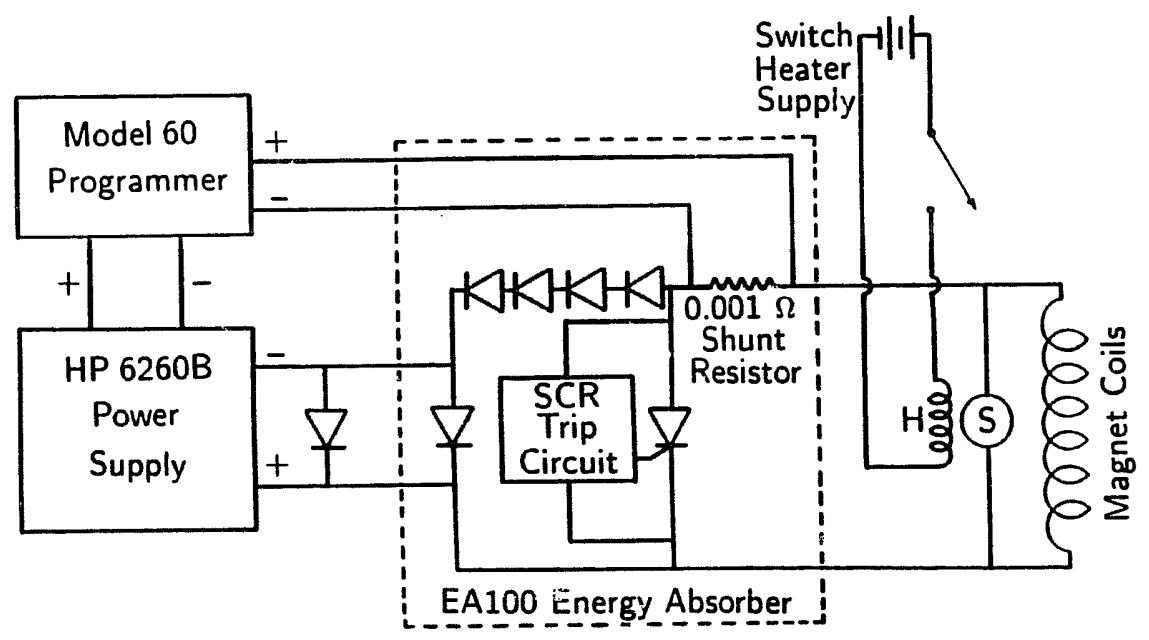

Fig. 12. Block diagram of magnet charging/discharge system. H denotes the persistent switch heater coil, and $S$ the superconducting persistence switch.

of the magnet power supply system is shown in fig. 12. The diode discharge unit flys aboard the payload, while the ramp controller and power supply are disconnected and removed prior to flight. The magnet persistent switch can be opened by radio command during flight to deactivate the magnet before recovery. A detailed account of magnet operation has been presented [18]. The magnet can produce a maximum field of $15 \mathrm{kG}$ at the center of a fiducial cube $20 \mathrm{~cm} \times 20 \mathrm{~cm} \times 20 \mathrm{~cm}$ located within the cylindrical room temperature bore of the cryostat. The fractional variation in $|\boldsymbol{B}|$ falls within the range $\pm 20 \%$ referenced to the value of $|B|$ at bore cenier. The cryostat holds 2901 of liquid helium, allowing the magnet to be operated for $\geq 100 \mathrm{~h}$ between cryogenic refills. The magnet and cryostat weigh $375 \mathrm{~kg}$.

The drift tube array consisted of $1.27 \mathrm{~cm}$ diameter spiral wound plastic tubes, with a nominal wall thickness of $30 \mu \mathrm{m}$, each with a $20 \mu \mathrm{m}$ diameter gold-plated tungsten wire strung down its center. Such tubes were originally developed for use as "vertex chambers" at colliding beam accelerator experiments and used to determine the position of particle interaction vertices to high spatial accuracy. The tube walls contributed 0.28 $\mathrm{g} / \mathrm{cm}^{2}$ of material for 24 planes of tubes totaling 323 in number. The 18 layers within the rigidity analysis portion of the array contained material (tube walls and gas) totaling $\sim 0.6 \%$ of a radiation length. The 216 "longitudinal" tubes were aligned parallel to $B$ in 16 layers, and performed the tracking measurements used to determine rigidity. This was accomplished by stopping a time-to-digital-converter (TDC) which had been started by the experimental trigger with the output of a discriminator triggered by the drift tube pulse. The timing start was provided by a logical coincidence between the TOF scintillators. Such a measurement records the time necessary for electrons to drift from the primary ionization track left by a particle to the wire. The distance of closest approach for the particle to the wire can be inferred from this time, once the time-to-distance mapping function known as the "time-to-space" function for the particular tube configuration, wire voltage, magnetic field, and gas mixture has been determined.

The geometrical arrangement for the longitudinal tubes is shown in fig. 13. High rigidity particles incident

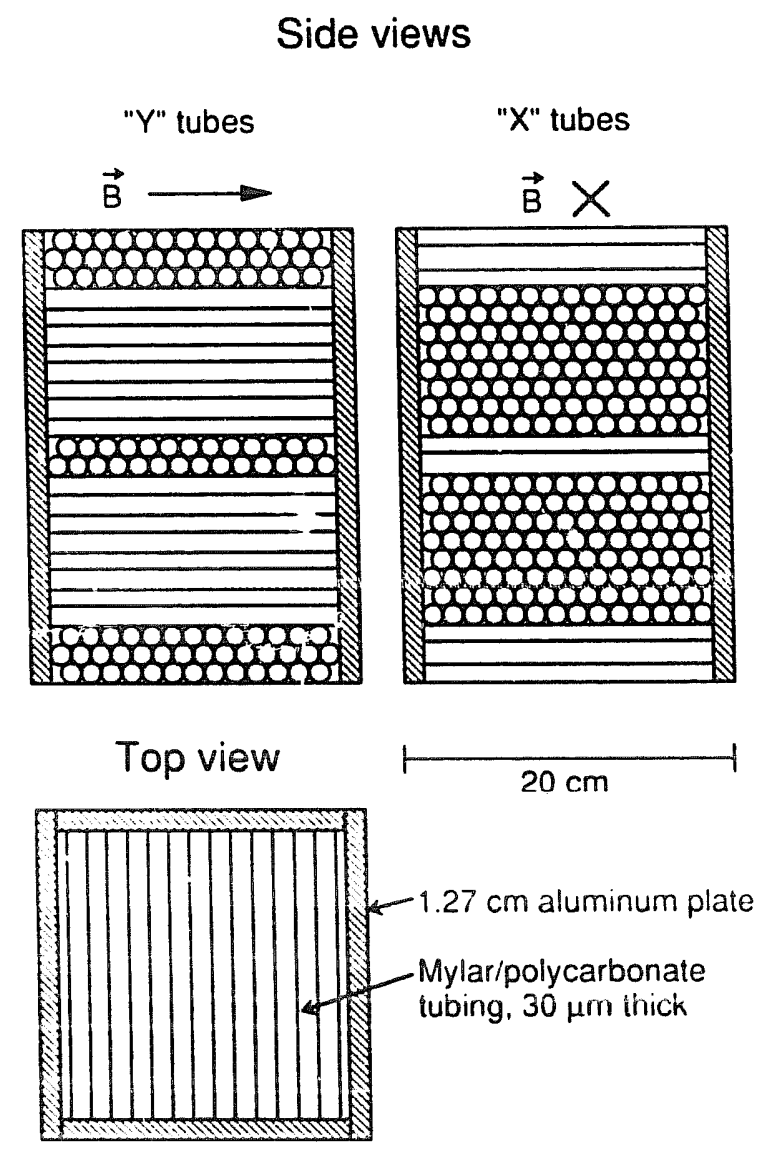

Fig. 13. Orthogonal views of the PBAR drift tube array. 
at $60^{\circ}$ do not produce ambiguous trajectory data, since particles at the same distance $b$ t on opposite sides of the wires in the top of the array hit different tubes in the bottom of the array. By spacing the two eight-tube groupings a non-integer number of wire separation distances apart, such ambiguities have been eliminated. The remaining 107 "transverse" tubes are normal to the field in eight layers and aid three-dimensional track reconstruction, as well as helping to reject large-angle scatterings within the array.

The tubes resided within four precision machined $1.27 \mathrm{~cm}$ thick aluminum plates which, in addition to holding the array accurately together, also served as gas flow manifolds. The tube and wire mount mechanics are shown in cross section in fig. 14. Precision machining techniques were employed throughout, resulting in an rms wire placement error of $30 \mu \mathrm{m}$ as determined from preflight muon tracking data. The development of the special thin-walled plastic tubing [19], as well as a detailed account of array assembly procedures, has been reported [18]. Of special note are the use of a conductive polycarbonate layer to ensure the integrity of the ground plane in the event that the cathode aluminizing were scratched, and the use of two types of epoxy [20] to attach the plastic tubing to the brass mounting plugs: Eccobond solder $57 \mathrm{C}$, a silver-loaded material for electrical contact; and Eccobond 45, a structural epoxy for hermetic gas seal. Both adhesives were applied simultaneously during drift tube assembly.
Several quality checks were performed on each tube prior to assembly of the array. The most important of these was a leak check, to ensure that each iube was gas-tight. This was crucial, since one severely leaking tube would interrupt the serial gas flow to the entire array. Each tube was pressurized to 2 psi while mounted in a special jig, and the pressure monitored for $1 \mathrm{~min}$ with a high precision pressure gauge. The accepted tolerance level of $\mathrm{d} P / \mathrm{d} t<0.01 \mathrm{psi} / \mathrm{min}$ ensured that the overall leak rate for the array would remain below $2 \times 10^{-2} \mathrm{l} / \mathrm{min}$ if all tubes leaked at this level. The great majority of tubes had no detectable leak rate. Tubes with leak rates greater than $0.01 \mathrm{psi} / \mathrm{min}$ were rebuilt. The end-to-end resistance for each tube was also checked to evaluate the quality of the electrical ground provided by the glue. The $1 \Omega$ standard deviation observed about the $14 \Omega$ mean indicated little variation in the electrical grounding of the tubes. Finally, the entire array was checked for gas leaks once the tubes had been mounted into the plates but before the wires had been strung.

The wires were strung at a nominal tension of $0.1^{\circ} \mathrm{N}$ by means of a $15 \mathrm{~g}$ weight passed over a low-friction pulley. The nominal sag for the gold-plated $20 \mu \mathrm{m}$ diameter tungsten wires [21] was $-2 \mu \mathrm{m}$ at this tension. The wires were anchored within the wire-mount ferrules by soldering, since mechanical crimping was difficult to accomplish and had proven unreliable in tests. Electrical contact was made via a gold-plated

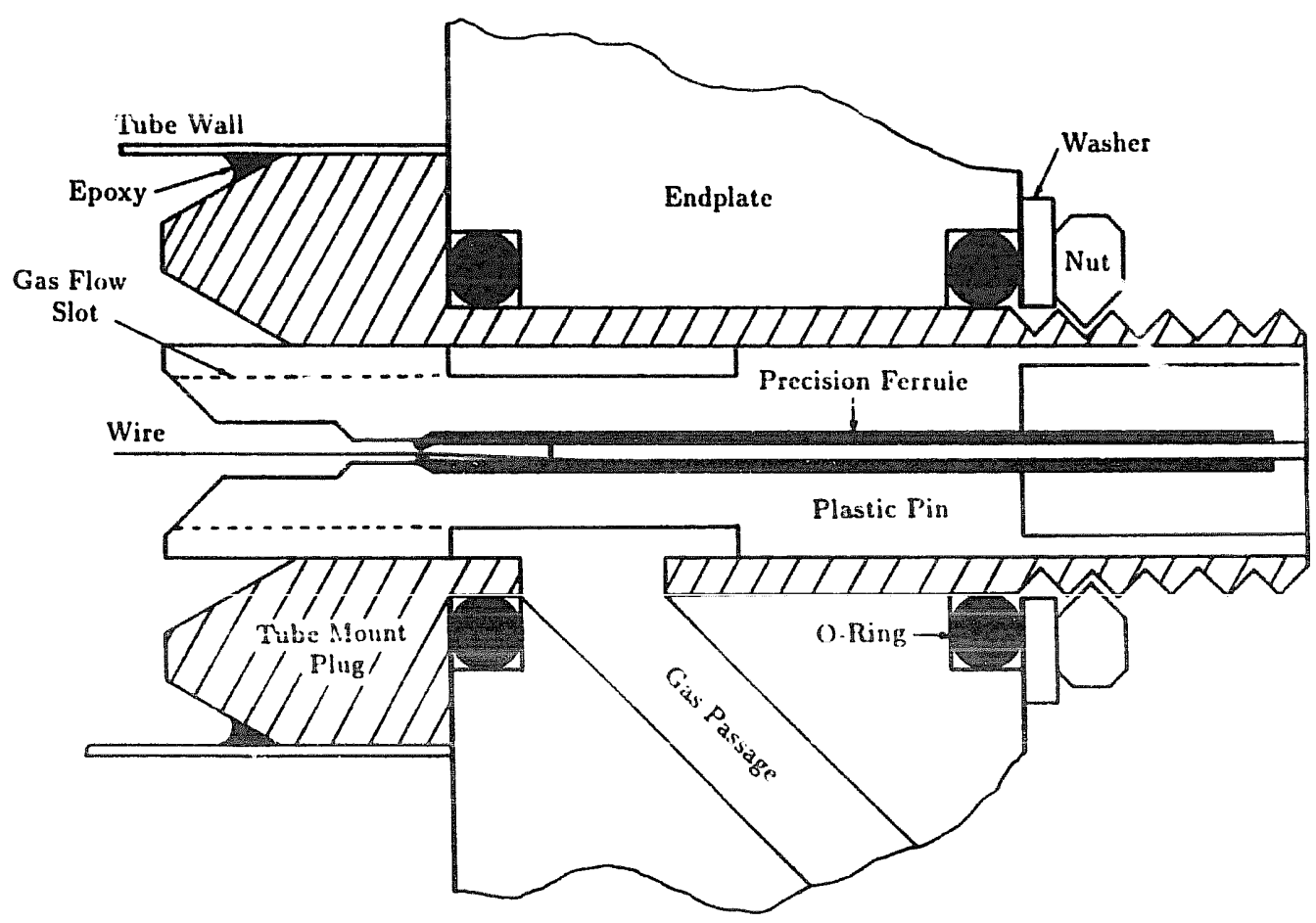

Fig. 14. Cross-sectional detail of drift tube and wire-mount mechanics. 


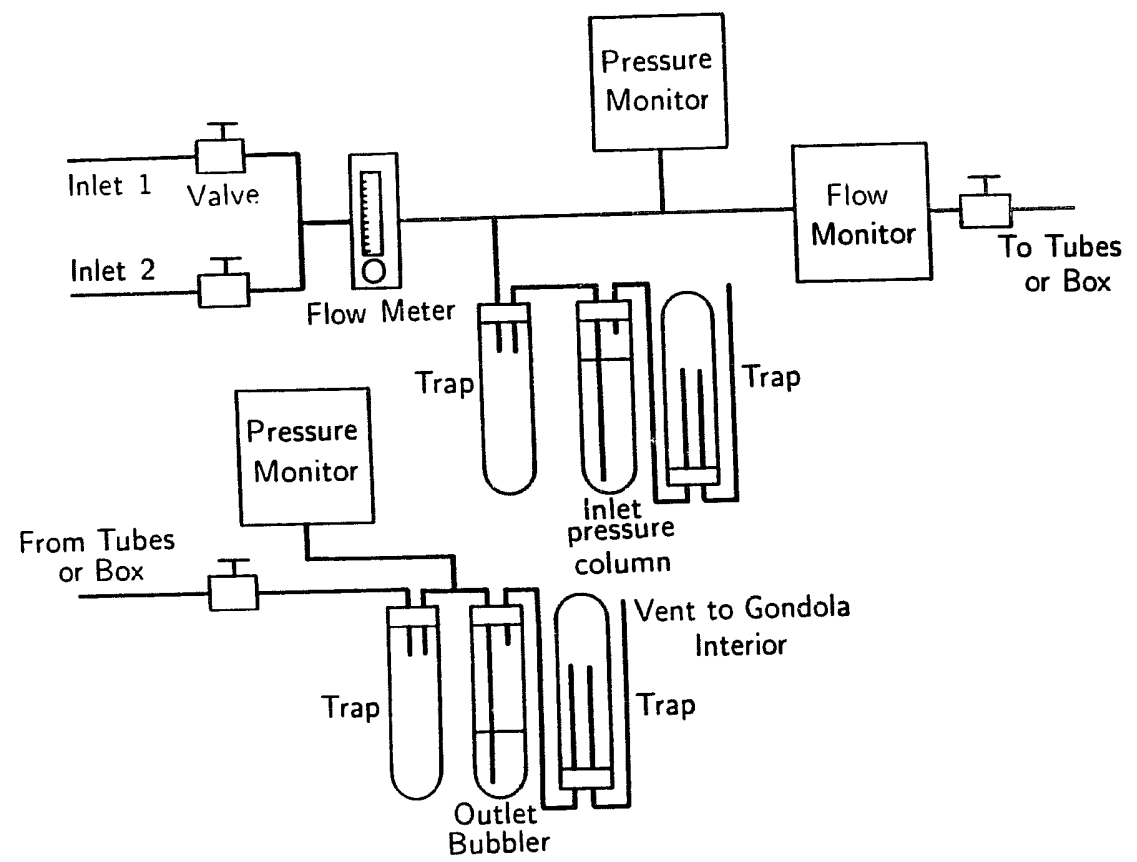

Fig. 15. Block diagram for the drift tube gas flow system. Two such identical systems were used: one to flow serially through the drift tubes and another to maintain a chamber gas atmosphere within the aluminum box surrounding the tubes. The bubbler and trap assemblies were constructed from glass test tubes, rubber stoppers, and glass tubing. Standard Swagelok gas fittings and tubing were used to distribute the gas. Overpressure was established by filling the bubblers with light mineral oil. The trap assemblies prevent the mineral oil from spilling into the gondola and flow system in the event that the payload tips over during recovery.

connector socket [22], since subsequent soldering would cause the wires to detach. The socket was part of a simple home-made coaxial connector used to connect the RG179 cable to the drift tubes.

A mixture of $50 \%$ argon-50\% ethane $\left(\mathrm{C}_{2} \mathrm{H}_{6}\right)$ flowed serially through the 323 tubes and maintained slightly above ambient pressure by a mineral oil column "bubbler" at a flow rate of $0.11 / \mathrm{min}$. A schematic of the flow system is shown in fig. 15. Two such systems were used. The top and bottom of the array were covered by $0.5 \mathrm{~mm}$ thick aluminum plates. These plates served both to protect the drift tubes and to seal the array sufficiently so that the argon-ethane mixture could be introduced into the inter-tube region within the plates. While not hermetic, this outer "chamber" formed by the mounting and cover piates was sufficientiy gas-tigint to allow the volume surrounding the tubes to be filled with drift tube gas and maintained at slight positive pressure relative to the ambient by a gas flow rate of $\sim 0.05$ $1 /$ min into the plates. Had a $t$,be wall ruptured, interrupting the serial gas distribution, the outer plates would continue to maintain an argon-ethane atmosphere within the tubes allowing normal operation. This has been dubbed the "nested leaky box" gas flow system, since neither the drift tubes nor the plates were perfectly gas-tight, although the tubes were nearly so. This precaution proved unnecessary as the array survived flight and recovery without rupturing.

\section{Water Cherenkov counter}

For the data reported in this work with $0.17 \leq \beta \leq$ $0.8, \beta$ has been obtained via TOF analysis. For higher velocities $\beta$ iniormation from both TOF and $\mathrm{CK}$ has been employed, each weighted by its variance. This technique has extended the data sample to include particles in the interval $0.8 \leq \beta \leq 0.93$.

The early PBAR design concept [23] called for a silica aerogel Cherenkov radiator in combination with an air-filled light integration box (LIB). Such a counter was experted to yieid $\sim 35$ photoelectrons for a minimum ionizing cosmic ray muon, with a threshold $\beta_{\mathrm{T}} \approx$ 0.9 . Subsequent difficulty in obtaining the aerogel (the factory producing the material was destroyed in an explosion) led to water as the chosen radiator. While the refractive index of water ( $n=1.37$ at $265 \mathrm{~nm}$ ) gives a lower value of $\beta_{\mathrm{T}}=0.73$, and hence more saturated response at higher values of $\beta$ where it contributes most to velocity measurement, the prospect of bette: photoelectron counting statistics partially outweighs this disadvantage. 
The construction of a radiator/LIB system employing water posed some unique engineering problems. Cherenkov counters used for velocity measurements in the PBAR energy range often employ roughened plastic radiators inside light integration boxes, the interiors of which have been coated with a diffuse reflective material such as barium sulfate $\left(\mathrm{BaSO}_{4}\right)$ [24]. Water has the unfortunate property of dissolving barium sulfate raint (which is formulated in aqueous solution [24]) as well as aluminum, used both for the construction of LIB and as a reflective coating. The use of extremely pure water in order to increase the absorption length exacerbates this problem. Chemical equilibrium between the ultrapure water and its container can only be established at the expense of dissolving the container into the water, degrading the reflective properties of the container walls and decreasing the absorption length of the water. Ultrapure water should therefore be segarded as a corrosive substance. In addition, LIB must be watertight and of great structural integrity, since the water radiator is quite heavy $(\sim 100 \mathrm{~kg})$ and subjected to a $10 \mathrm{~g}$ loading at parachute deployment. The final configuration solved these problems to produce a counter of superb spatial uniformity with a yield of $\sim 220$ photoelectrons for a minimum ionizing cosmic ray muon. The counter employed no waveshifters and detected primary Cherenkov photons. Since the spectral distribution of primary Cherenkov photons emitted per unit path length per unit wavelength is inversely proportional to wavelength squared $\left(\mathrm{d} N /(\mathrm{d} \lambda \mathrm{d} x) \sim \lambda^{-2}\right)$ it is imperitive that the water radiator have the longest possible ultraviolet (UV) attenuation length and that the diffuse reflector lining the light integration region have the highest possible UV reflectance. Tests of a prototype counter as well as detailed response calculations aimed at optimizing these two properties have been previously reported [25.26].

Fig. 16 depicts the mechanical design of LiB and its magnetic shielding. The walls were inclined $35^{\circ}$ from the vertical, forming the sides of a truncated octagonal pyramid. The water radiator was $15 \mathrm{~cm}$ thick throughout. Four RCA 8854 PMTs with gallium phosphide ("quantacon") first dynodes viewed the radiator through water-filled tubular lightpipes. These lightpipes set each tube back $20 \mathrm{~cm}$ from the wall of LIB to allow magnetic shields to extend past the photocathode by the same amount. Two-layer magnetic shields covered each tube and interlocked with the wall of LIB for mechanical integrity. The two-stage shield design employed a 5 $\mathrm{mm}$ thick, low permeability $(\mu)$ steel outer layer which resists magnetic saturation, covering a high $\mu$ inner shield of $1 \mathrm{~mm}$ thick $80 \%-20 \%$ nickel-iron alloy to provide sufficient attenuation of the magnet fringe field, in the same manner as the TOF shields described in section 4. These shields allowed the PMiTs to be operated in proximity to the magnet without significant degradation of their performance in fields of $\sim 250 \mathrm{G}$. The PMT voltage divider circuit is shown in fig. 17. Zener diodes were employed so that the total cathode to anode high voltage could be varied without affecting the gain of the first dynode stage, which is crucial in determining the single photoelectron resolution. The 15 $\mathrm{M} \Omega$ load resistor caused some saturation of the photo-
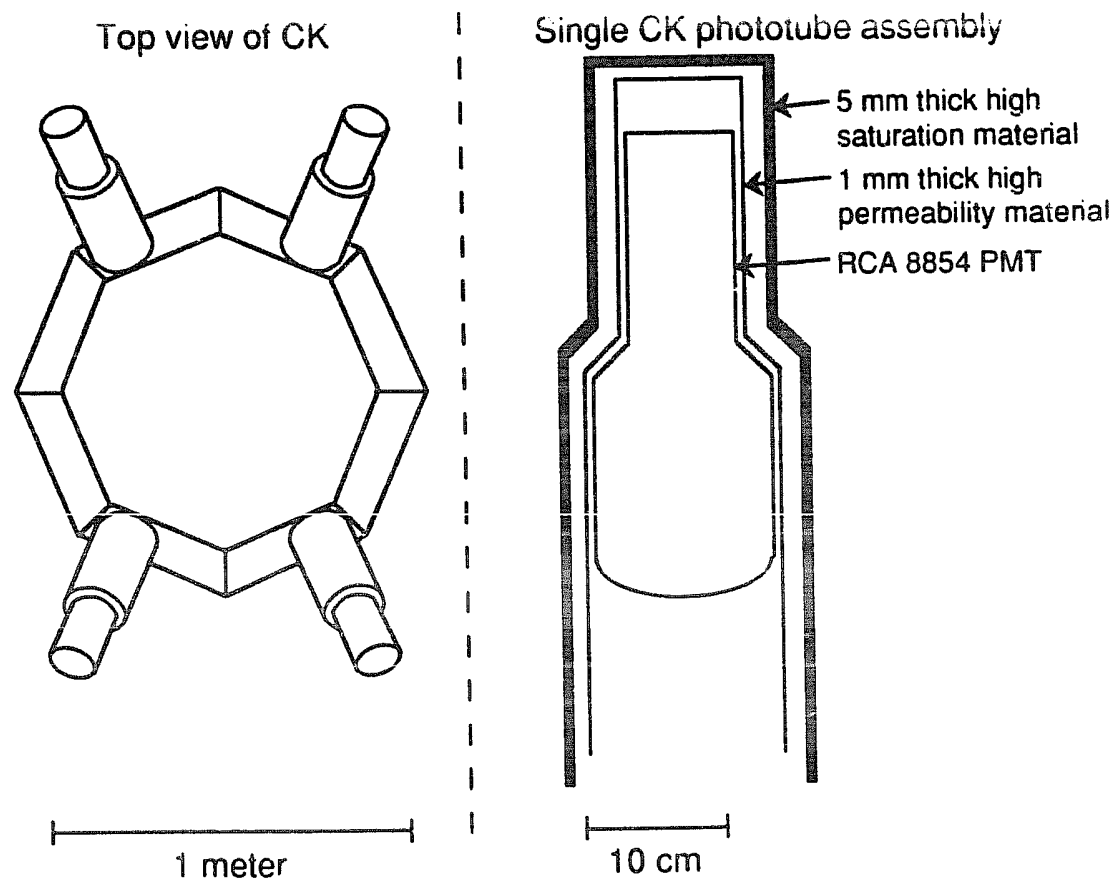

Fig. 16. Mechanical layout of the Cherenkov counter and detail of magnetic shielding. 


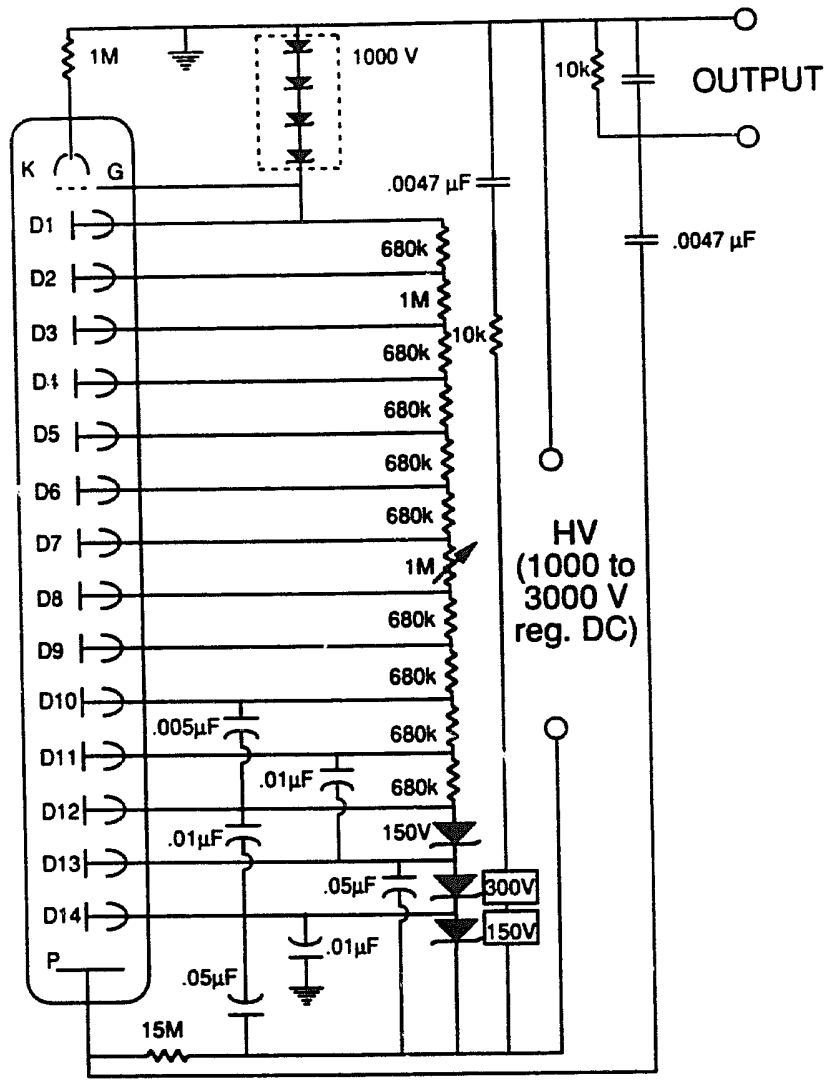

Fig. 17. Schematic diagram of Cherenkov photomultipler tube high voltage divider and signal network.

tube gain characteristic at high light levels, and should be replaced with a $0.5 \mathrm{M} \Omega$ value for improved dynamic range and high rate performance.

The development of a diffuse reflector which could tolerate immersion in highly pure deionized-distilled water was crucial, and involved testing several compounds. Ultimately, blocks of specially sintered white Halon polytetrafluoroethylene (PTFE) plastic $\sim 0.06$ $\mathrm{cm}$ thick were chosen to line the integration region of
LIB, while transparent Teflon aluminized on one side lined the lightpipes with the aluminizing away from the water radiator. PTFE powder has been shown [27] to be more reflective than barium sulfate powder in the UV, with comparable reflectance in the visible. The Halon plastic material was made by heating powdered raw material to a specified temperature for a specified period of time in an oven ("sintering"). This process causes the powder grains to fuse together and form a white plastic. Commercially available sintered sheets of compressed PTFE come in "commercial" and "virgin" grades. Their reflectances, as measured with a Cary 14 spectrophotometer using barium sulfate powder as a standard, are plotted in fig. 18. Even the virgin grade material is too tightly compressed, making it translucent and reducing both its overall reflectivity and diffusive properties. To obtain the required quality, we performed all the scintering operations to produce the material lining the counter. The "O-Halon" (octagon liner) curve in fig. 18 is for material made by packing Ausimont USA brand G-80 PTFE resin to a density of $1 \mathrm{~g} / \mathrm{cm}^{3}$ between glass sheets and heating in a ceramics kiln at a temperature of $355-375^{\circ} \mathrm{C}$ for $1 \mathrm{~h}$. The temperature ramp-up rate was 4-6 h, with a cool-down time of 8-19 h for various batches of material. The reflectance of this material is comparable to that of barium sulfate powder (and hence superior to that of barium sulfate paint), within the $\sim 1 \%$ error in the spectrophotometer measurements. This material was used to line the PBAR Cherenkov counter. To ensure the highest possible reflectance, the Halon lining the counter was sanded several days prior to flight to remove any surface contamination which may have accumulated. Based on a computer model of the LIB configuration, a bulk reflectance $\sim 1.5 \%$ worse than tha: obtained from the spectrophotometer measurements was deduced for the Halon liner flown aboard PBAR. This was most likely due the way the Halon was mounted within LIB. The restricted size of the available scintering oven required tha? the Halon be produced in

\section{Reflectance of CK Liners}

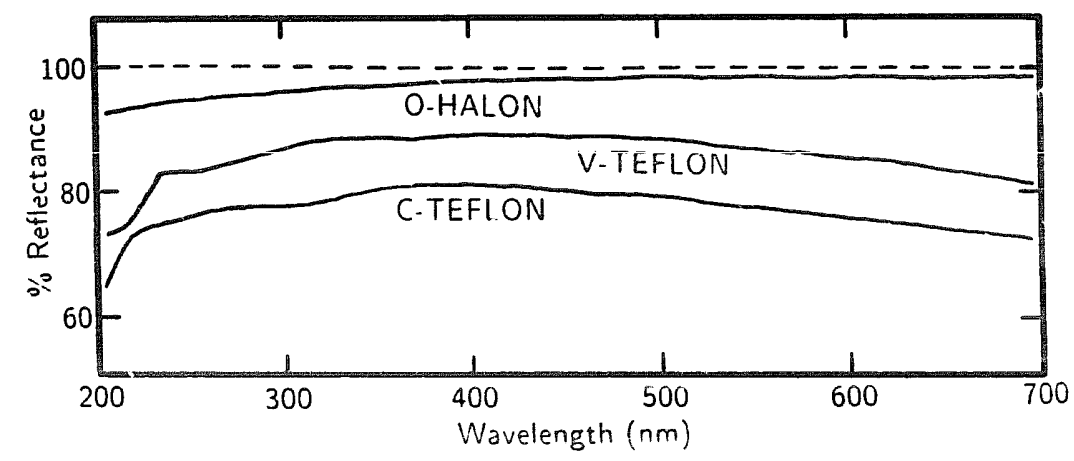

Fig. 18. Reflectance versus wavelength for three diffuse reflectors tested for possible use in the PBAR Cherenkov counter: commercial grade Teflon (C-Teflon), virgin grade Teflon (V-Teflon), and a high quality batch of Halon ( $\mathrm{O}$-Halon) used to line the PBAR Cherenkov counter. 
multiple "tiles" of various shapes, typically several hundred square centimeters in area. Some light could be lost through the seams between the tiles, effectively lowering the bulk reflectance of the liner.

The attenuation length of the water radiator, and hence the total number of photoelectrons collected for a minimum ionizing particle, depends critically on water purity. Three possible purification techniques were evaluated: distilled water, deionized water, and distilled deionized water. Of these, distilled deionized water was found to be the best, and adopted as the flight radiator. This water was the output product of a Corning Megapure water purification system consisting of an organic filter, an inorganic filter, and an all-glass distillation apparatus. This self-contained system was transported to the field and used to prepare fresh water shortly before flight. The purified water was stored in new polyethelene bottles prior to introduction into the counter. Some evidence was observed for a decrease in attenuation length by a factor of $\sim 2$ for water stored in bottles for a period of two months. For best performance it is important to purify and install the water radiator as close to flight as practical.

\section{Balloon flight}

The instrument was housed in a spherical aluminum gondola shell of thickness $-0.84 \mathrm{~g} / \mathrm{cm}^{2}$ covered by an
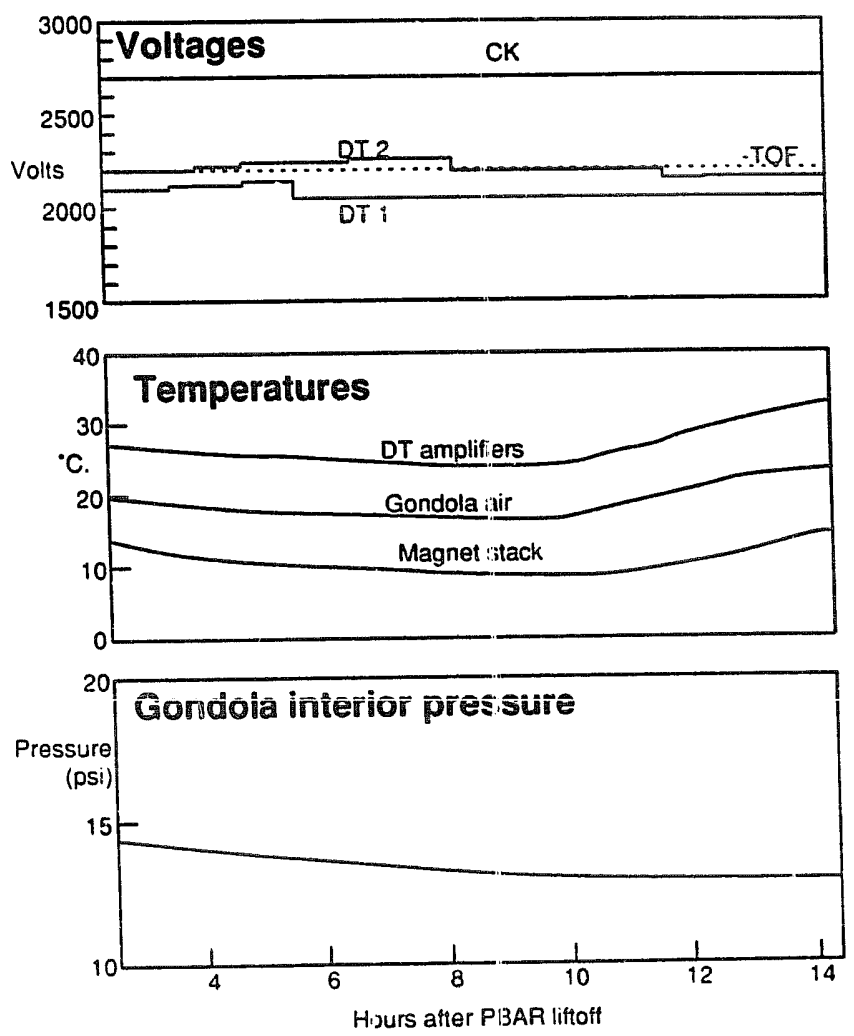

Fig. 19. Temperatures, gonidola pressure, and instrument operating voltages from the housekeeping, data taken during flight. insulating urethane foam layer $-4 \mathrm{~cm}\left(0.3 \mathrm{~g} / \mathrm{cm}^{2}\right)$ thick painted white. The final spectrometer configuration had an aperture of $72 \mathrm{~cm}^{2} \mathrm{sr}$ as determined by Monte Carlo calculation using the method of Sullivan [28]. The magnet was charged to a current of $47.8 \mathrm{~A}$ to produce a central field of $8.75 \mathrm{kG}$ and locked into persistent mode. PBAR was launched from Prince Albert, Saskatchewan on the evening of August 13, 1987 at 2:02:50 Universal time (8:03 PM local time). The package was lofted by a $7.9 \times 10^{5} \mathrm{~m}^{3}$ helium-filled balloon. The payload consisted of the $1568 \mathrm{~kg}$ PBAR package with an additional $341 \mathrm{~kg}$ of balast. The flight achieved a float altitude of $36 \mathrm{~km}$ corresponding to 4.8 $\mathrm{g} / \mathrm{cm}^{2}$ of residual atmosphere above the payload for a duration of $10.5 \mathrm{~h}$, of which $7.6 \pm 0.8 \mathrm{~h}$ was actual data acquisition "live time". At float the latitude varied between $53^{\circ} 18^{\prime} \mathrm{N}$ and $54^{\circ} 23^{\prime} \mathrm{N}$, while the longitude varied between $104^{\circ} 55^{\prime} \mathrm{W}$ and $108^{\circ} 04^{\prime} \mathrm{W}$. The environment within the gondola was excellent during flight, as illustrated in fig. 19. The gondola air temperature varied between $16.5^{\circ} \mathrm{C}$ and $22.5^{\circ} \mathrm{C}$. The internal pressure decreased $\sim 10 \%$ from 0.980 to $0.877 \mathrm{~atm}$.

\section{Data analysis}

This section describes the techniques employed io extract particle properties from the raw flight data. Further detail can be found in Lowder [29] and Bower [26]. In addition, Tomasch [18] has presented detailed mean response calculations for the instrument.

The on-board data acquisition system sent down 52 16-bit words of data per event frame, to which the ground computer appended an additional 5 data words as the incoming data were written to tape. "Housekeeping" frames were sent once every $6 \mathrm{~s}$ to monitor instrument operating parameters, as well as the gondola environment. Both "low sensitivity" and "high sensitivity" ADC signals from the TOF and Cherenkov phototubes were obtained by the use of passive splitters to increase the dynamic range of these counters. For TOF the high sensitivity signal was 32 times the low sensitivity signal. while for CK the ratio was $21: 1$. With the exception of calibration events, the TOF and DT data were zero suppressed. Only TOF tubes for which a nonzero ADC value was registered were recorded, while only those drift tubes which registered a hit were recorded. TDCs which overflowed were also suppressed. All CK ADCs were read out for each event.

\subsection{Rigidity analysis}

To reject sevure scatterings and events with multiple particles, as well as to suppress extraneous tube hits which sometimes accompanied otherwise valid drift tube 
data, the following drift tube filter algorithm was applied:

1) Define the tubes parallel to $\boldsymbol{B}$ ("longitudinal" tubes) as $\mathrm{X}$ tubes. These are arranged in two groups of eight layers. The remaining three groups of tubes normal to $B$ (transverse tubes) are denoted as $\mathrm{Y}$ tubes (see fig. 13). An event is accepted if six of eight tubes in each $\mathrm{X}$ group were hit and at least one tube in each $\mathbf{Y}$ group was hit.

2) Take those $Y$ tubes with no other hits in the same layer and fit a straight line to their centers. If less than two layers have a single hit tube the event is rejected.

3) Throw out any $Y$ tube which is more than one tube diameter away from the line ed in step 2 .

4) Reject the event if there does not remain at least one hit tube in each of the three $Y$ sections.

5) Repeat steps 2 and 3 for the top eight-layer section of $\mathrm{X}$ tubes.

6) Reject the event if the top $X$ section no longer contains six layers wit's sits.

7) Repeat steps 5 and 6 for the bottom eight-layer section of $\mathbf{X}$ tubes.

Once the events had been filtered, the "hit radii" or "impact parameters" (the distance of closest approach for the trajectory relative to the wire in a given tube) were determined. This process was only done for the $\mathbf{X}$ tubes (i.e. the plane normal to $B$ where curvature is observed). Hit radius determination required the following steps:

1) Calculation of the actual electron drift time from the raw measured drift time.

2) Caiculation of the impact parameter from the timeto-distance mapping relation or "time-to-space function" obtained from a combination of theory and measured gas properties.

3) A successive approximation procedure to improve the parameters of the electron drift model in step (2) and fine tune the timing offsets.

To obtain the true electron drift time several offsets must be added to the raw time interval measured in flight. This is illustrated in fig. 20 . If the time at which the particle passes through a drift tube is denoted as $T_{0}$ then the start and stop times are

$T_{\text {stop }}=T_{0}+T_{\text {drift }}+T$ (DT cables and electronics),

$$
\begin{aligned}
T_{\text {start }}= & T_{0}+\operatorname{TOF}\left(z_{\mathrm{DT}-\mathrm{S} 2} / z_{\mathrm{S} 1-\mathrm{S} 2}\right)+(50 \mathrm{~cm}+x) / v_{\text {eff }} \\
& +T(\text { light guide })+T(\mathrm{PMT} \text { transit }) \\
& +T(\text { TOF cables and electronics })
\end{aligned}
$$

from which it follows that

$$
\begin{aligned}
T_{\text {drift }}= & T_{\text {meas }}+\operatorname{TOF}\left(z_{\mathrm{DT}-\mathrm{S} 2} / z_{\mathrm{S} 1-\mathrm{S} 2}\right) \\
& +x / v_{\text {eff }}+T_{\text {scint }}-T_{\mathrm{DT}},
\end{aligned}
$$

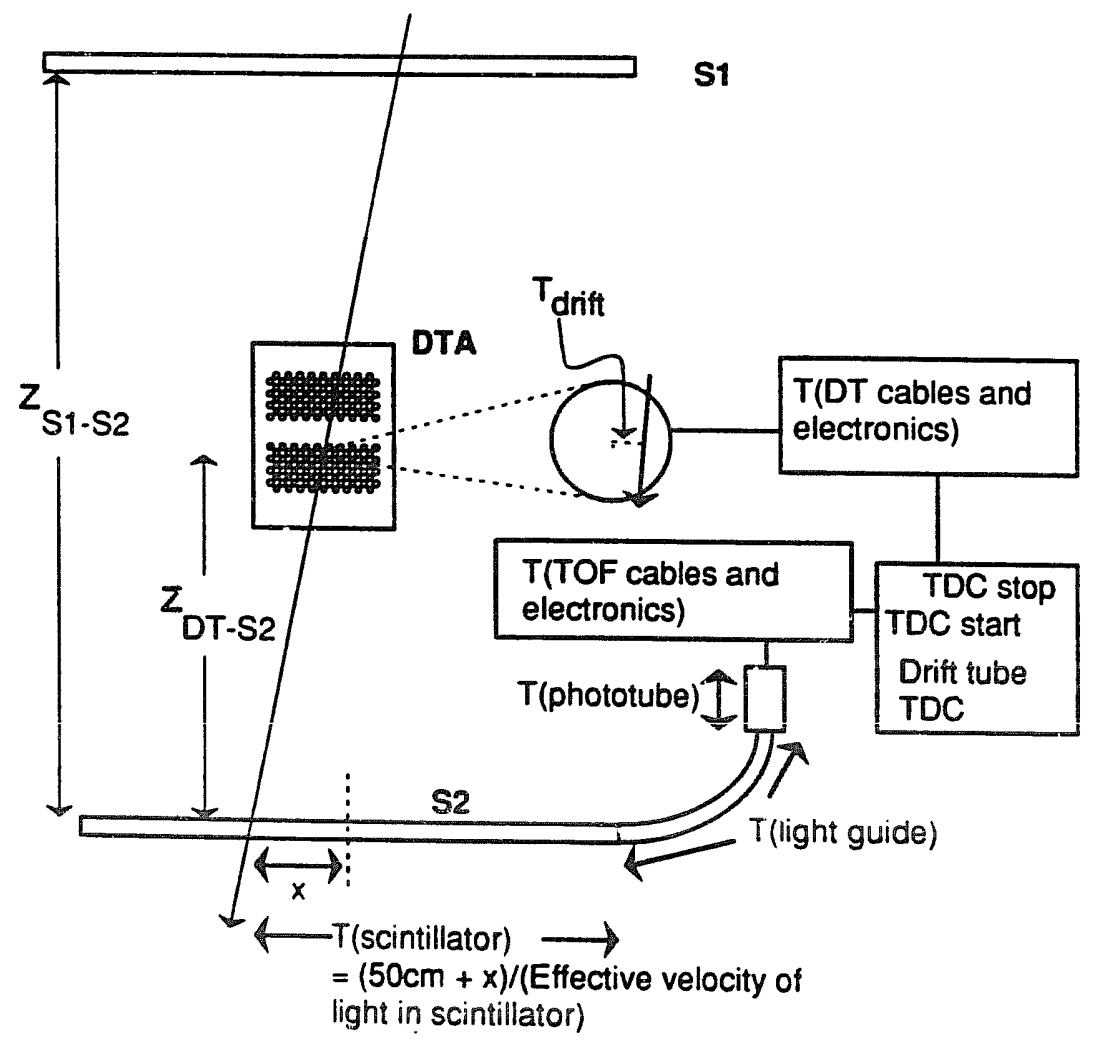

Fig. 20. Diagram showing various timing offsets that must be added to and subtracted from the time interval measured by the arift tube TDC to ohtain the actual electron drift timse. 
where the disiances $z_{\mathrm{DT}-\mathrm{S} 2}, z_{\mathrm{S} 1-\mathrm{S2}}$, and $x$ are defined in fig. 20 . The quantity $v_{\text {eff }}$ is the effective propagation speed of light in the scintillator. The first three terms of eq. (12) are specific to a given event. $T_{\text {scint }}$ combines all the offsets due to signal propagation through the TOF scintillator and electronics from the scintillator midpoint and depends only on which of the bottom scintillators is hit. $T_{\mathrm{DT}}$ is the sum of the delays in the drift tube cables and electronics. This can be obtained from a histogram of all the drift times recorded for a given tube. Such a histogram shows a uniform distribution between two time values, the lower corresponding to zero drift time (events at the wire), the higher corresponding to the maximum drift time (events at the tube wall).

Once the drift time had been extracted, the impact parameter or hit radius $h$ was obtained from the timeto-space relation

$T_{\mathrm{drift}}=\int_{r_{0}}^{h} \frac{\mathrm{d} r}{w_{\mathrm{r}}(r)}$

where $w_{\mathrm{r}}$ is the radial drift speed of electrons at a distance $r$ from the wire and $r_{0}$ is the radius at which avalanche multiplication begins near the wire. The drift speed is a function of the electric field, gas pressure, and magnetic field. The magnetic field was assumed to be parallel to the wires, and a field map was calculated from an accurate knowledge of the current distribution in the magnet coils. Tube voltages and gas pressure were known from housekeeping data, and the electric field was readily calculated from Gauss's law for two concentric cylinders. The measured zero-field drift speed [30] was then corrected for $E \times B$ effects using the method reported by Baranko et al. [31].

To obtain the best possible position resolution, the timing offsets for each drift tube had to be iteratively adjusted to minimize the tracking residuals. Hit radii were calculated using the best first guess for the timing offsets and momentum fits performed for each event. The residual, the difference between the closest approach of the fitted trajectory and the actual hit radius, was determined for each tube in an event. For each tube these residuals were binned and an average residual calculated. The timing offsets for each tube were adjusted by an amount proportional to the average residual for that tube. The adjustment was scaled so that a
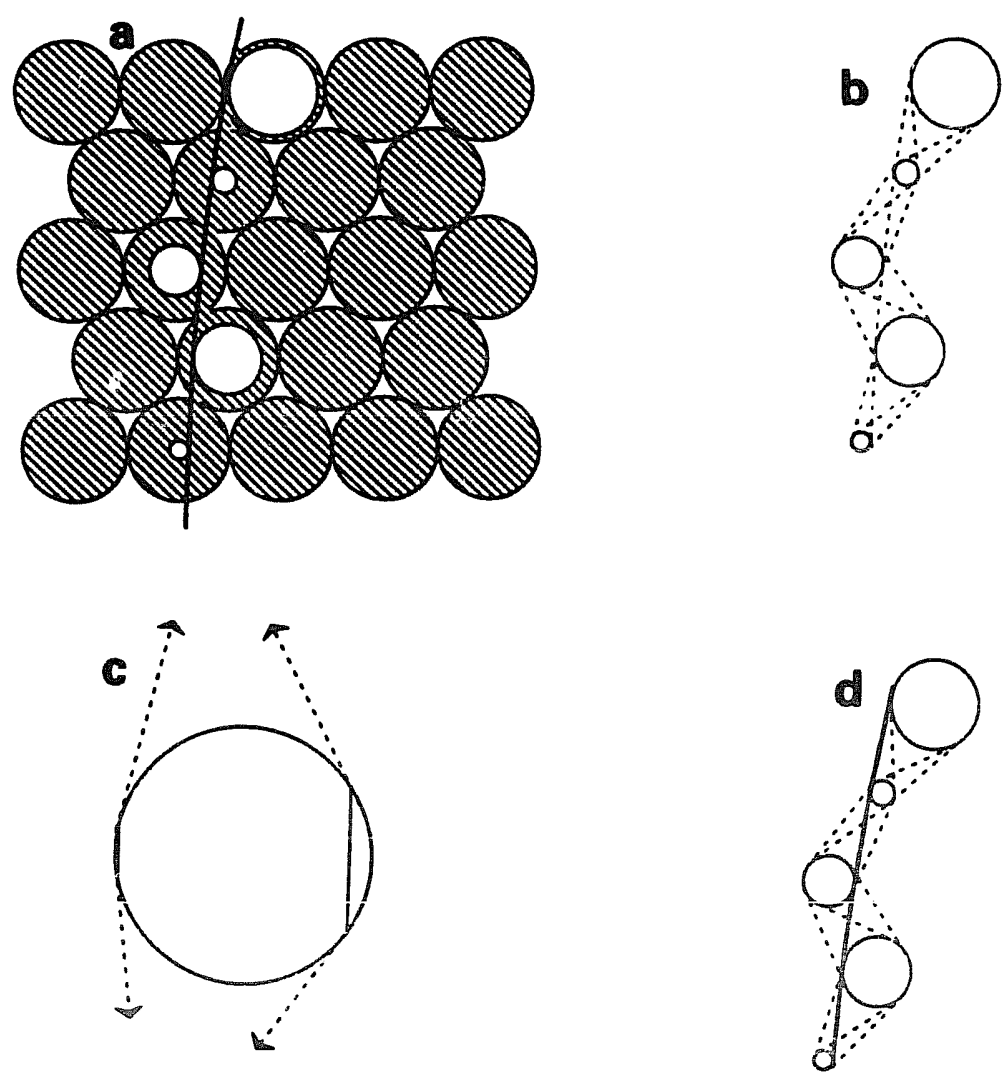

Fig. 21. Summary of track finding algorithm. (a) Graphic representation of drift tube data. Drift tubes are shaded: the white circles appear in hit tubes with a white circle's radius equal to that tube's hit parameter. (b) The analysis software calculates the tangeni lines between adjacent pairs of circles. (c) The basic method: on a given circle, the tangent chords pointing to the previous circle are paired with those pointing to the next circle, and the pair with the shortest distance between their end points on the given circle are chosen. In the case illustrated above, the pair of tangents on the left would be chosen. (d) In practice, several adjacent pairs of circles are considered at orice, and the path is chosen that minimizes the sum of the end-point distances. 
hit radius $50 \mu \mathrm{m}$ too small would receive a $1 \mathrm{~ns}$ increase in timing offset (this corresponds roughly to the distance electrons drift in 1 ns in the gas). This was repeated several times until the residuals showed no more appreciable change.

The drift tube impact parameters do not give the particle trajectory directly, but instead define a set of circles to which the trajectory must be tangent. To trace the path of the particle and identify its proper orientation relative to the circles required the development of a "track finder" algorithm, illustrated in fig. 21. Each pair of adjacent circles has four common tangent chords, as shown. For a given circle, the algorithm picks out the common tangents with the preceding and succeeding circles which are closest together on the circle under consideration.

Once the points along the particle path had been defined by the track finder, the particle rigidity was determined from a fit to these points using a two-dimensional modification of the method of Wind [32]. This method was checked against the CERN routine MOMENTM, which implements Wind's method in three dimensions, with good agreement between the two routines. After the fit, any tube exhibiting a residual from the fitted trajectory of greater than $350 \mu \mathrm{m}$ was removed and the event refit.

\subsection{TOF analysis}

To determine a particle's velocity from the TOF timing data required the following steps:

1) For events where an interaction or shower occurred and more than one S1 or S2 scintillator were hit, the drift tube tracking data were used to select which slabs were traversed by the particle observed in the hodoscope. The event was rejected unless both phototubes on the chosen $\mathrm{S} 1$ and $\mathrm{S} 2$ scintillators had valid $A D C$ and $T D C$ information. In the following discussion the four chosen phototubes are labeled 1 , 2, 3, 4 with 1 and 2 referring to the S1 tubes and 3, 4 to the $\mathrm{S} 2$ tubes.

2) Each of the four TDC values was corrected for "timing walk" using the tube's ADC value.

3) Time of flight was determined as

$$
\begin{aligned}
\text { TOF }= & 0.5\left(\mathrm{TDC}_{3}+\mathrm{TDC}_{4}\right) \\
& -0.5\left(\mathrm{TDC}_{1}+\mathrm{TDC}_{2}\right)-T_{\text {offset }}
\end{aligned}
$$

where $T_{\text {offset }}$ is the net timing offset due to electronic delays and cable lengths for the particular combination of $\mathrm{S} 1$ and $\mathrm{S} 2$ counters hit.

4) $\beta=\frac{1.5 \mathrm{~m}}{c \text { TOF } \cos \theta_{\text {zenith }}}$.

Timing walk corrections were necessary due to the variation of TOF PMT signal amplitudes relative to the fixed discriminator thresholds. This correction was of the form

$\mathrm{TDC}_{\mathrm{cor}}=\mathrm{TDC}_{\mathrm{raw}}-a(\mathrm{ADC})^{b}$,

where the parameters $a$ and $b$ were found by searching the $a \times b$ parameter space using events with high rigidity $(\beta \sim 1)$ so as to minimize the width of the TOF $\cos \theta_{\text {zenith }}$ distribution. For TDC and ADC in units of electronic channels ( $50 \mathrm{ps} /$ channel and 0.25 $\mathrm{pC} /$ channel respectively) the values $a=200$, and $b=$ 0.25 yielded the narrowest timing residuals. This form of the timing walk correction yielded distributions $20 \%$ narrower than those obtained for more conventional parameters where $b$ is fixed at 0.5 [33]. Once the timing walk corrections were applied, $T_{\text {offset }}$ was determined for each $\mathbf{S} 1-\mathrm{S} 2$ combination by making a histogram of TOF $\cos \theta_{\text {zenith }}$ and taking $T_{\text {offset }}$ as the difference between the peak value and $5 \mathrm{~ns}$, the correct value for a $\beta=1$ particle traversing $1.5 \mathrm{~m}$.

\subsection{Charge analysis}

The sense of curvature in the magnetic field determines the sign of the particle charge. The light output
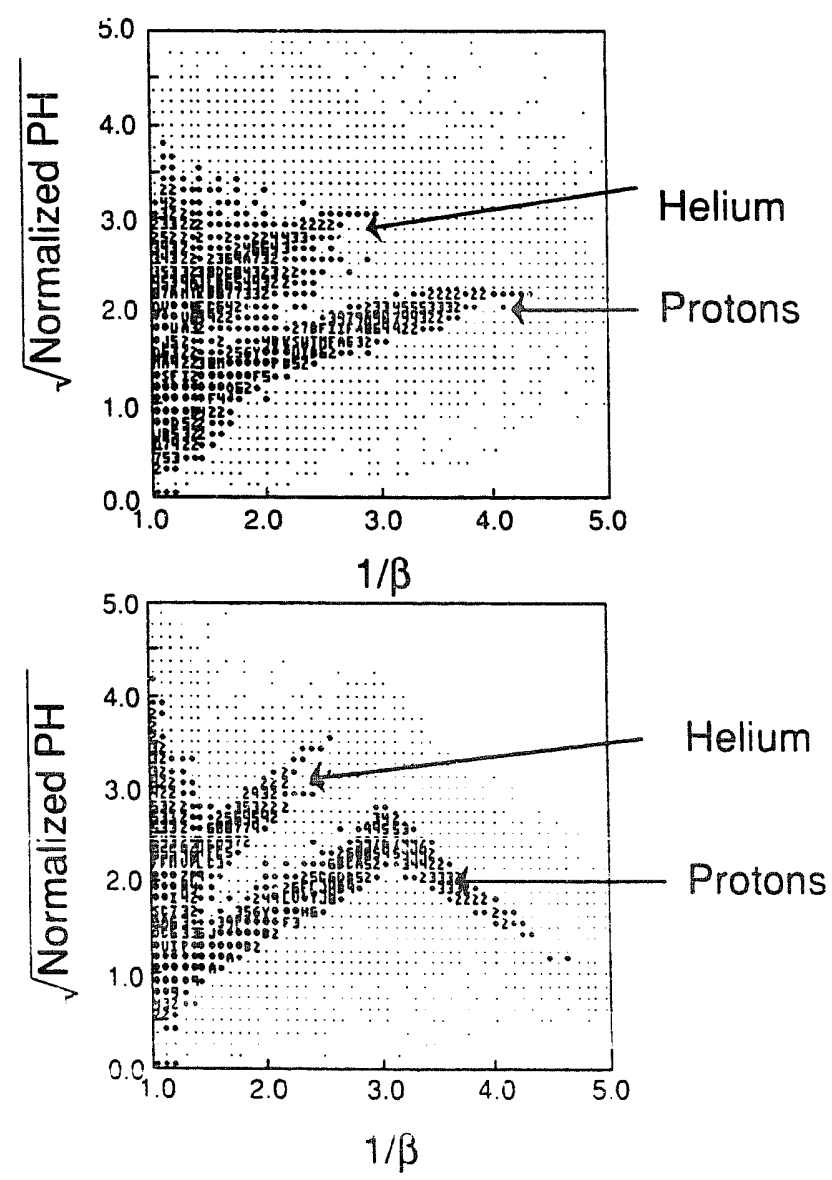

Fig. 22. Scatter $p: n t$ of scintillator signals versus $1 / \beta$ showing clearly resolved charge tands in both $\mathrm{S} 1$ and $\mathrm{S} 2$ counters. 


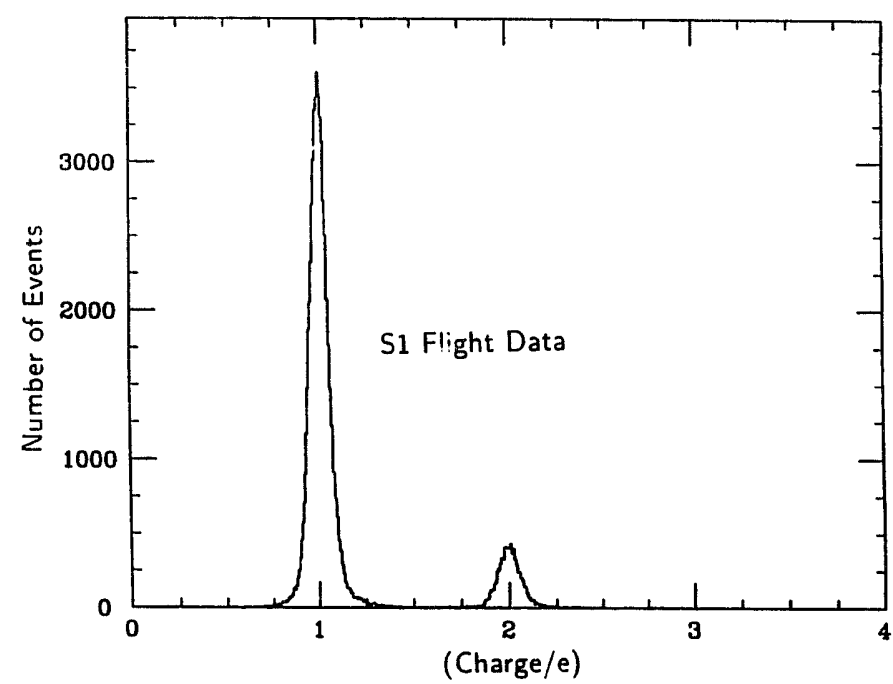

Fig. 23. S1 charge histogram for flight data.

in the scintillators, and hence the scintillator pulse heights, are proportional to $(z / \beta)^{2}$ for $(z / \beta)<10$, permitting the magnitude of charge to be determined from the pulse height data. The ADC values were pedestal subtracted and the different PMT ADC values normalized to correct for differences in gain, scintillator response, and light collection efficiency. This was accomplished by using signals from minimum ionizing particles as a standard and assigning the same number of normalized channels to the $z=1, \beta=1$ peak for each ADC. Once normalized, the quantity (ADC1 $A D C 2)^{1 / 2}$ was calculated as the average signal for a given S1 module, since this quantity minimizes the dependence of the signal on position. The charge for a given event was determined by dividing the normalized S1 signal by the expected S1 signal for a $Z=1$ particle with the same $\beta$ as inferred from plots of S1 signal versus $\beta^{-1}$. This vependence was also checked against mean response values calculated from energy loss and tabulated scintillator response characteristics [18] and found to be in excellent agreement. Fig. 22 displays scatter plots of pulse height versus $\beta^{-1}$ for S1 and S2, clearly showing the separating of protons and helium. Charge histograms are reported in fig. 23. Finally, fig. 24 reports the charge resolution obtained as a function of $\beta$ for the flight data.

\subsection{Cherenkov analysis}

If a particle does not slow appreciably within the counter, its velocity can be readily calculated for a known Cherenkov signal CK by solving the FrankTamm expression (8) multiplied by the thickness of radiator traversed by the particle to obtain

$\beta=\frac{1}{n} \sqrt{\frac{\mathrm{CK}_{\max } Z^{2}}{\mathrm{CK}_{\max } Z^{2}-\mathrm{CK} \cos \theta_{\text {zenith }}}}$,

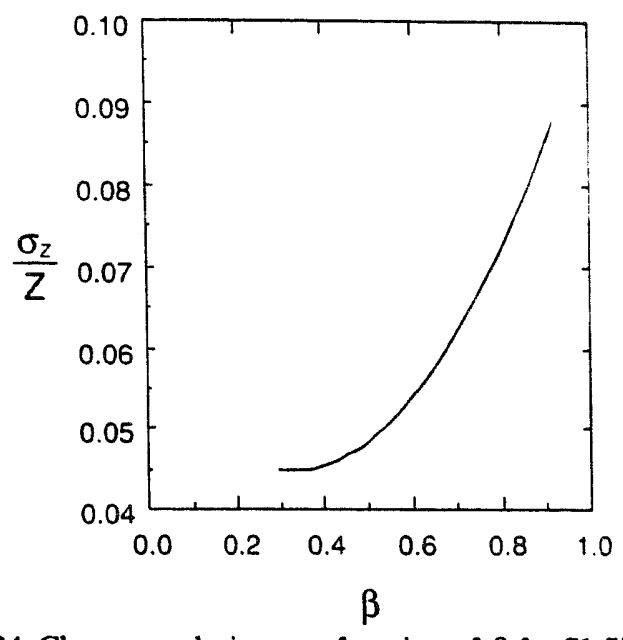

Fig. 24. Charge resolution as a function of $\beta$ for $S 1$ flight data.

where $\mathrm{CK}_{\max }$ is the signal for a $Z=1, \beta=1$ particle at vertical incidence and $n$ is the refractive index. Due to the large load resistor used to protect the CK PMTs from high currents, there was saturation of the signals at high light levels. The values of $\mathrm{CK}_{\max }$ and $n$ were obtained from a fit to $C K \cos \theta_{\text {zenith }}$ versus $\beta_{\text {TOF }}^{-2}$ (shown in fig. 25), which should be linear for a given $z$. The departure from linearity above $\beta=0.9$ is due to phototube saturation as discussed in section 6 . To correct for
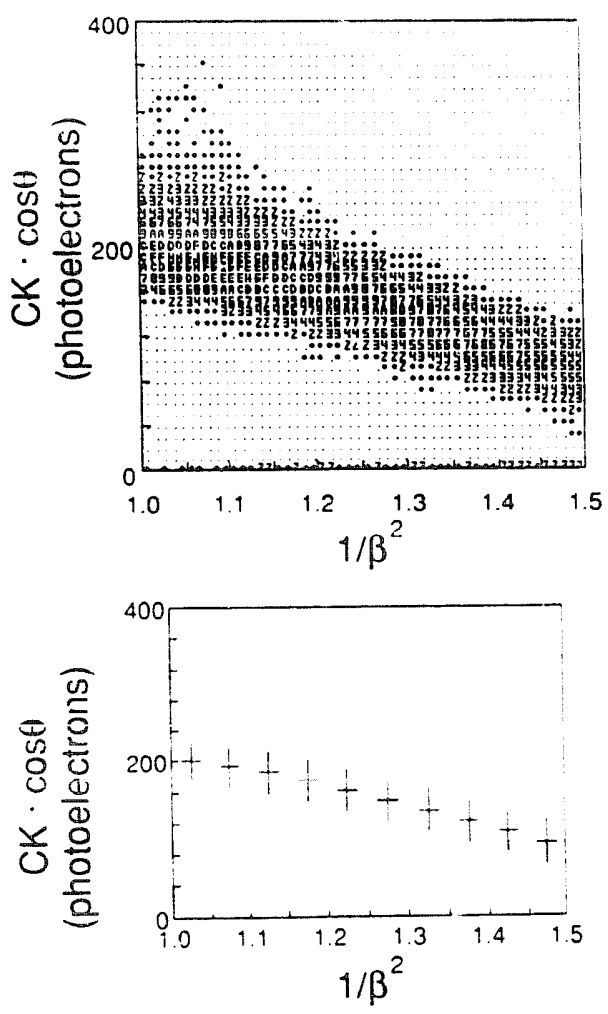

Fig. 25. Scatter plot of $\mathrm{CK} \cos \theta$ versus $1 / \beta^{2}$ for flight data. The plot of mean values illustrates the deviation from linearity due to phototube saturation. 
this, the parameters $\mathrm{CK}_{\max }$ and $n$ were defined differently over two separate velocity intervals. For $\beta>0.828$ (partially saturated regime) the best agreement between the TOF and CK velocity determinations was obtained for $\mathrm{CK}_{\max }=222$ and $n=1.369$. For $\beta<0.828$ (no saturation) the values $\mathrm{CK}_{\max }=229$ and $n=1.377$ yield the best agreement, for both atmospheric muons measured before flight and flight data. The mean velocity in CK deduced from eq. (16) was corrected to account for slowing in CK to yield the velocity within the magnet bore. This was done emperically by means of a quadratic function relating $\beta_{\text {TOF }}$ and $\beta_{\mathrm{CK}}$ with coefficients determined from a fit to the data. This correction was only applied for $\beta<0.828$, since above this value proton slowing within CK can be neglected.

\section{Instrument performance}

\subsection{Rigidity spectrometer}

Fig. 26 demonstrates the drift tube resolution attained for protons in the interval $0.17 \leq \beta \leq 0.8$ during flight. The abscissa reports residuals from fitted quadratic trajectories for all 216 longitudinal tubes, with no cuts imr sed on incident particle zenith angle. The resulting histogram is nearly perfectly Gaussian in character. The value $\sigma_{1 \text { tube }}=110 \mu \mathrm{m}$ from the peak full width at half maximum ( $\sigma=$ FWHM. $/ 2.36)$ represents an average over all tubes, energies, and incident zenith angles. This illustrates one of the inherent advantages of drift tubes over multiwire chambers: their resolution does not degrade with increasing zenith angle.

From the discussion in section 1 it follows that the drift tube resolution contributes a sagitta error

$\delta S_{\text {track }} \sim \frac{3}{\sqrt{2 \times 16}} 110 \mu \mathrm{m} \sim 58 \mu \mathrm{m} ;$
$\left(\frac{\delta S}{S}\right)_{\text {track }} \approx\left(4 \times 10^{-3} \%\right)(R / \mathrm{MV})$.

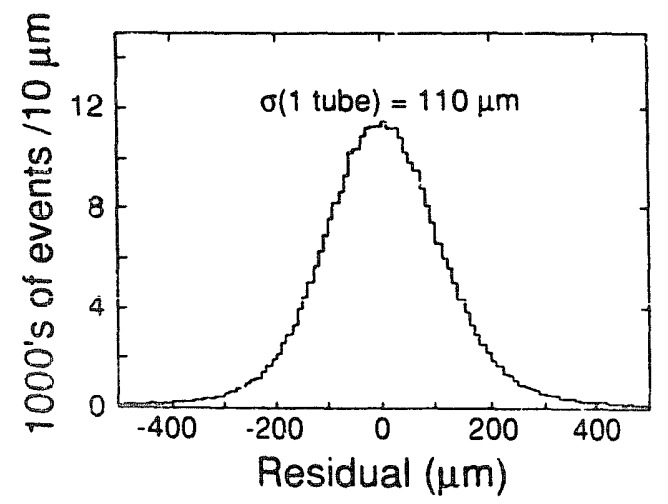

Fig. 26. Histograrn of residuals for drift tube measurements about the fitted trajectories for flight data. The width of the ti..ribution implies a single tube resolution of $110 \mu \mathrm{m}$ averageat over all rigidities. zenith angles and tubes parallel to $B$ for $0.17<\beta<0.8$.
From eq. (5) the sagitta for a $\beta=0.93$ proton (the smallest sagitta for data discussed in this work) is -0.5 $\mathrm{mm}$, so the tracking errors are expected to contribute $(\delta R / R)_{\text {track }}<10 \%$. The sagitta error due to multiple scattering can be estimated as $\Delta S_{\mathrm{MCS}} \approx S_{\text {plane }}^{\mathrm{rms}}=$ $(l /(4 \sqrt{3})) \theta_{0}$ from the simple Gaussian approximation [34] to multiple scattering where:

$\theta_{0}=\frac{14.1(\mathrm{MeV} / c)}{p \beta} z \sqrt{\frac{L}{L_{\mathrm{R}}}}\left[1+\frac{1}{9} \log _{10}\left(\frac{L}{L_{\mathrm{R}}}\right)\right]$.

In eq. (17) $L / L_{\mathrm{R}}$ is the number of radiation lengths of material traversed, with $\theta_{0}$ expressed in rad. For the drift tube array this works out to be:

$\theta_{0} \approx \frac{0.86}{\beta R}$,

$\delta S_{\mathrm{MCS}} \approx \frac{2.48 \mathrm{~cm}}{\beta R}$,

$\left(\frac{\delta S}{S}\right)_{\mathrm{MCS}} \approx \frac{1.7 \%}{\beta}$,

for $R$ in MV. At $\beta=0.93$ multiple scattering contributes $\delta S \sim 12 \mu \mathrm{m}$ for protons, somewhat iess than the error introduced by tracking. At $\beta=0.17$ (the lowest $\beta$ allowed by the analysis cuts) the contribution is $\delta S_{\text {MCs }}$ $-900 \mu \mathrm{m}$ which amounts to $(\delta R / R)_{\mathrm{MCS}}-10 \%$ when referenced to the $9 \mathrm{~mm}$ sagitta expected for a proton with this $\beta$. Since absolute sagitta error due to tracking remains at the $60 \mu \mathrm{m}$ level independent of $\beta$, the multiple scattering contribution to momentum error dominates tracking error over the range $0.17 \leq \beta \leq 0.6$. This illustrates one reason why the array has been designed to incorporate a minimum amount of matter: to preserve adequate rigidity resolution at low velocities.

Based on the foregoing discussion, one might argue that DT achieved higher tracking resolution than necessary since multiple scattering, not tracking error, limited momentum resolution at low velocities. Fig. 27 demonstrates why tracking errors must be as small as possible. The questior is not one of momentum resolution, but background rejection. Protons which experience single hard scatters will mimic particles with negative rigidity. This is traditionally referred to as "spillover" and constitutes a source of background inherent in all magnetic spectrometers. Qualitatively, fig. 27 illustrates that a large enough number of accurate measurements will distinguish a hard scatter trajectory from a curved one. To reject spillover, the number of points sampled along the trajectory must be large enough, and each such sampling accurate enough, so that the reduced $x^{2}$ for a hard scatier trajectory consisting of two straight lines meeting at a vertex will be higher when fitted incorrectly by a smooth quadratic curve than that for a true curved trajectory with the same sagitta. Thus, hard scatter events will populate the tail of a reduced $x^{2}$ distribution and can be cut from the analysis sample by a 


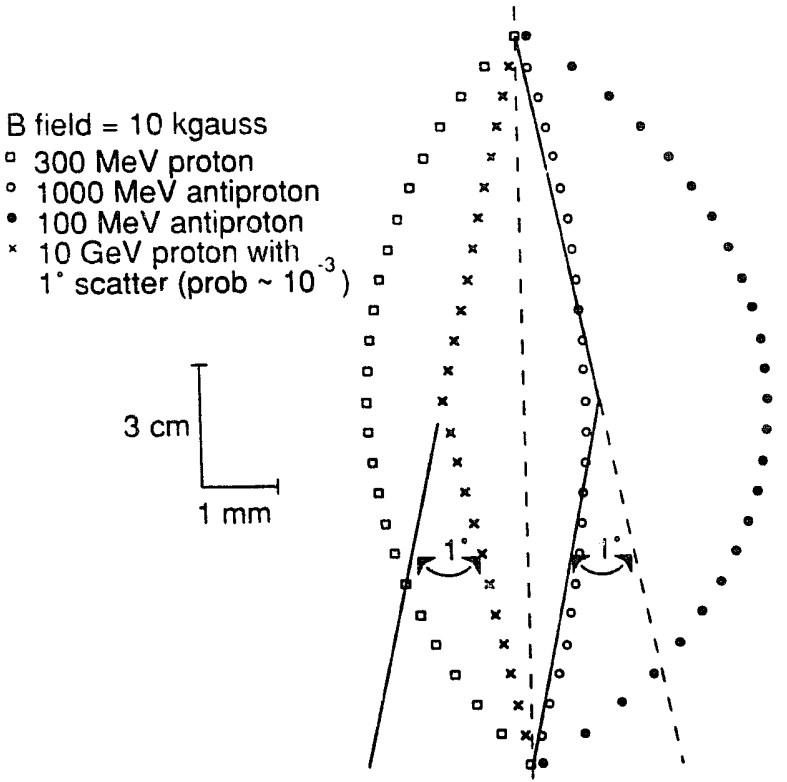

Fig. 27. An illustration of why many successive position measurements are necessary to reject scattering as a source of background to the antiproton search. Shown are antiprotons and protons, as well as a high energy proton scattering so as to mimic an antiproton.

"goodness-of-fit" cut $\chi^{2}<\chi_{0}^{2}$. This combination of low mass (to reduce the absolute number of hard scatters). high measurement density (24 total planes), and high resolution, all with an eye toward reducing scattering background, prompted the nickname "anti-vertex chamber" for DT, since particle interaction vertices were identified and rejected.

For $N$ measured points along the trajectory $(x, y)$. $i=1, \cdots, N$. the fitted trajectory is defined as $v_{i}^{\text {fit }}=$ $f\left(x_{i}\right)$. For a single tube resolution of $\sigma$ the parameter

$\mathrm{SS}=\sum_{i=1}^{N} \frac{\left(y_{i}-f\left(x_{1}\right)\right)^{2}}{\sigma^{2}}$

will be distributed with a $\chi^{2}$ distribution. For PBAR we defined the "goodness-of-fit" parameter $\chi_{R}$ (reduced $x)$, where

$\chi_{\mathrm{R}}=\sqrt{\sum_{i=1}^{N} \frac{\left(y_{i}-f\left(x_{i}\right)\right)^{2}}{N=3}}=\frac{\sigma}{\sqrt{N-3}} \sqrt{\mathrm{SS}}$.

which neaks at the value o $[(N-4) /(N-3)]^{1 / 2}$ and takes into account the three degrees of freedom lost in the fit to the trajectory. Fig. 28 shows the $\chi_{k}$ distr bution for protons in flight. The peak value of $110 \mu \mathrm{m}$ is in exact agreement with the value obtained from the width of the drift tube residual distribution shown in fig. 26 . By visually scanning negative rigidity events, the cut shown was determined to be the least stringent that could be placed on $\chi_{\mathbb{R}}$ to eliminate spillover background. Fig. 29 illustrates a typical spillover proton

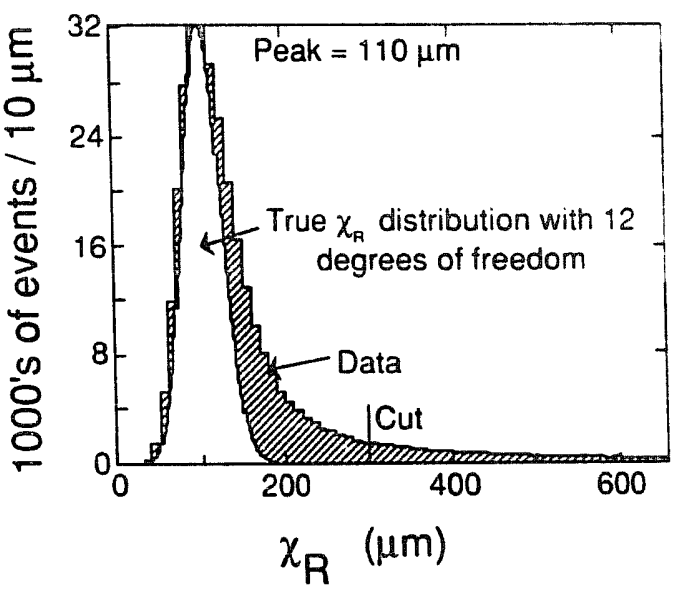

Fig. 28. Histogram of the drift tube goodness-of-fit parameter $\chi_{R}$, with a superimposed plot of the expected distribution for 12 degrees of freedom ( 15 tubes hit) and a single tube dispersion $\sigma=110 \mu \mathrm{m}$. Also shown is the cut made to eliminate scattering in the low velocity $(0.17<\beta<0.8)$ antiproton search.

rejected by this cut. The cut removed $\sim 10 \%$ of the proton sample and completely eliminated hard scatter events. Had the number of tube planes been fewer, or the single tube resolution worse, a larger fraction of the events would have been lost. For a small enough number of tubes, or for poor enough resolution, it becomes impossible to impose a $\chi_{R}$ cut and spillover events become antiproton candidates. This is dramatically il-

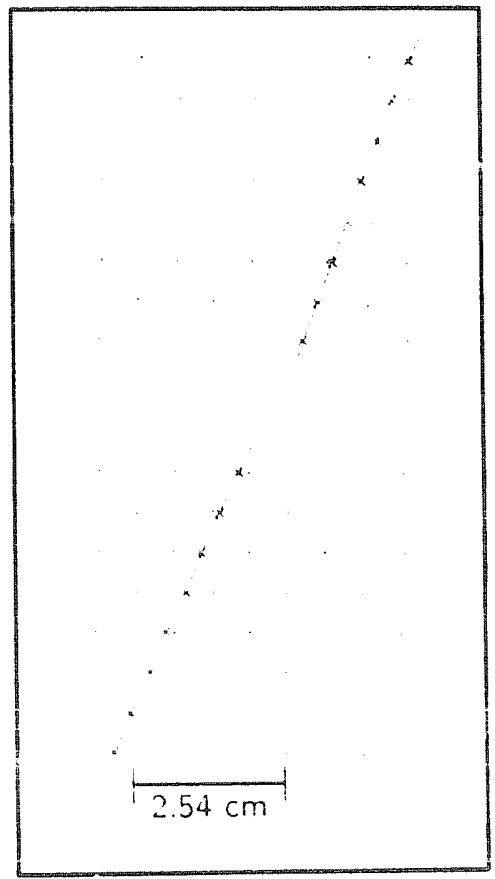

Fig. 29. A hard scalter event removed from the data sample by the cut shown in fig. 28. Although the event mimics a negative rigidity when fit, the top and bottom portions of the trajectory exhibit curvature corresponding to positive rigidity. This is readily observed by viewing the figure at an oblique angle. 

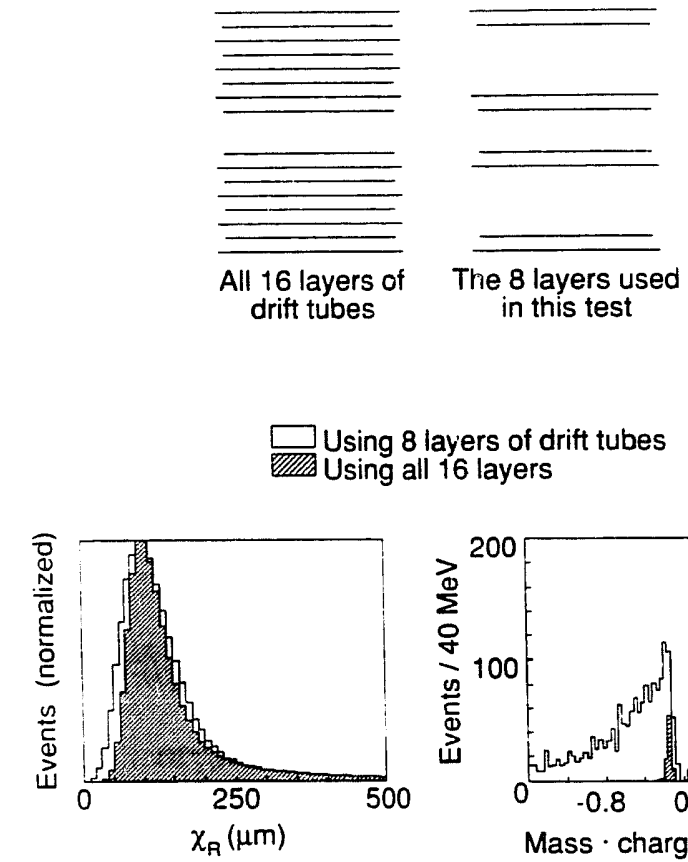

Using 8 layers of drift tubes Using all 16 layers

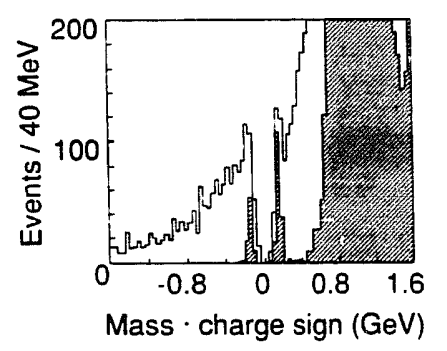

Fig. 30. The effect of removing 8 of the 16 drift tube layers from the analysis. Even though DT resolution remains unchanged, severe background due to large-angle scattering is introduced.

lustrated in fig. 30 , which demonstrates the severe background introduced if only eight drift tube planes are used for the rigidity analysis with all analysis cuts remaining unchanged.

\subsection{TOF resolution}

To determine the TOF resolution from flight data the technique illustrated in fig. 31 was emplcyed. The difference between the times recorded by the two PMTs on a given scintillator module is directly related to the location along the scintillator at which the farticle hit. A scatter plot of the position along the scintillator (obtained by the accurate extrapolation of the drift tube trajectory) versus this timing difference yields a linear relation with a slope corresponding to the effective speed of light propagation in the scintillator $v_{\text {eff }}$. By defining $\tan \theta$, the "track slope" as shown $n$ fig. 32. the TOF timing resolution can be extracted viıt the relation

$\sigma_{\mathrm{IOF}}=\left(\frac{L}{v_{\mathrm{eff}}}\right) \sigma_{\Delta \tan \theta}$

where $\Delta \tan \theta=\left(\tan \theta_{\mathrm{TOF}}-\tan \theta_{\mathrm{DT}}\right)$ is the difference between the slope determined from TOF and DT information. Since the error in slope as determined from the drift tubes is negligible compared to that derived from the PMT timing difference for the $\|$ bes at opposite ends of the scintillator module. the width of the $\Delta \tan \theta$ distribution shown in fig. 33 is due entirely to TOF fluctuations and implies an in-flight value $\sigma_{\text {TOF }}=160$ ps for $0.17<\beta<0.8$. The timing resolution as a function of $\beta$ is shown in fig. 34. The rapid increase below $\beta=0.35$ is due to particles ranging out in S2 and producing less light. The gradual increase above $\beta=0.35$ stems from the fact that scintillation output depends on $z / \beta$. The resolution extrapolated to $\beta=1$ is $185 \mathrm{ps}$ for $Z=1$ particles. Resolution under 100 ps was obtained for low velocity He events [29].

\subsection{Cherenkov resolution}

The Cherenkov detector exhibited spatial response variations of about $\pm 5 \%$ over its active area, with little correlation between enhanced light collection and proximity to phototube windows. The standard deviation for the CK signal was typically 26 photoelectrons and did not scale with the square root of the signal as expected, due to saturation and radiator thickness variation. The performance was still excellent, providing substantial improvement in mass resolution in the region $0.8<\beta<$ 0.93 . As shown in fig. 35 , CK provided the best velocity determination in this interval. The collection of 220 photuelectrons per minimum ionizing particle represents an outstanding level of efficiency for the collection of primary Cherenkov photons. The spatial uniformity was also excellent. Both of these qualities were a direct consequence of the care taken to optimize the clarity of the water radiator and the reflectivity of the Halon liner.

\section{O. Mass resolution}

Fig. 36 illustrates the overall fractional mass resolution obtained from the PBAR spectrometer system as a function of $\beta$. The actual resolution obtained (triangles) is compared to that predicted (solid curve) from the contributions made by $\mathrm{CK}$ velocity resolution $(\mathrm{CK})$, TOF velocity resolution (TOF), multiple Coulomb scattering (MCS), and drift tube tracking resolution (DTR). The performance at low velocities indicates that the simple Gaussian formulation of multiple Coulomb scattering used (yielding pure $\beta^{-1}$ dependence) is a poor approximation at low velocities. but yields conservative estimates.

Fig. 37 displays the mass histograms obtained from the flight data for the low velocity $(0.17<\beta<0.8)$ and high velocity $(0.8<\beta<0.93)$ analyses. The complete list of cuts placed on the data have heen reported [7]. The low velucity analysis used only TOF information to determire velocity, while the high velocity analysis employed both TOF and CK velocity determinations, each weighted by its respective variance, to arrive at a velocity for mass calculations. Protons and both positive and 

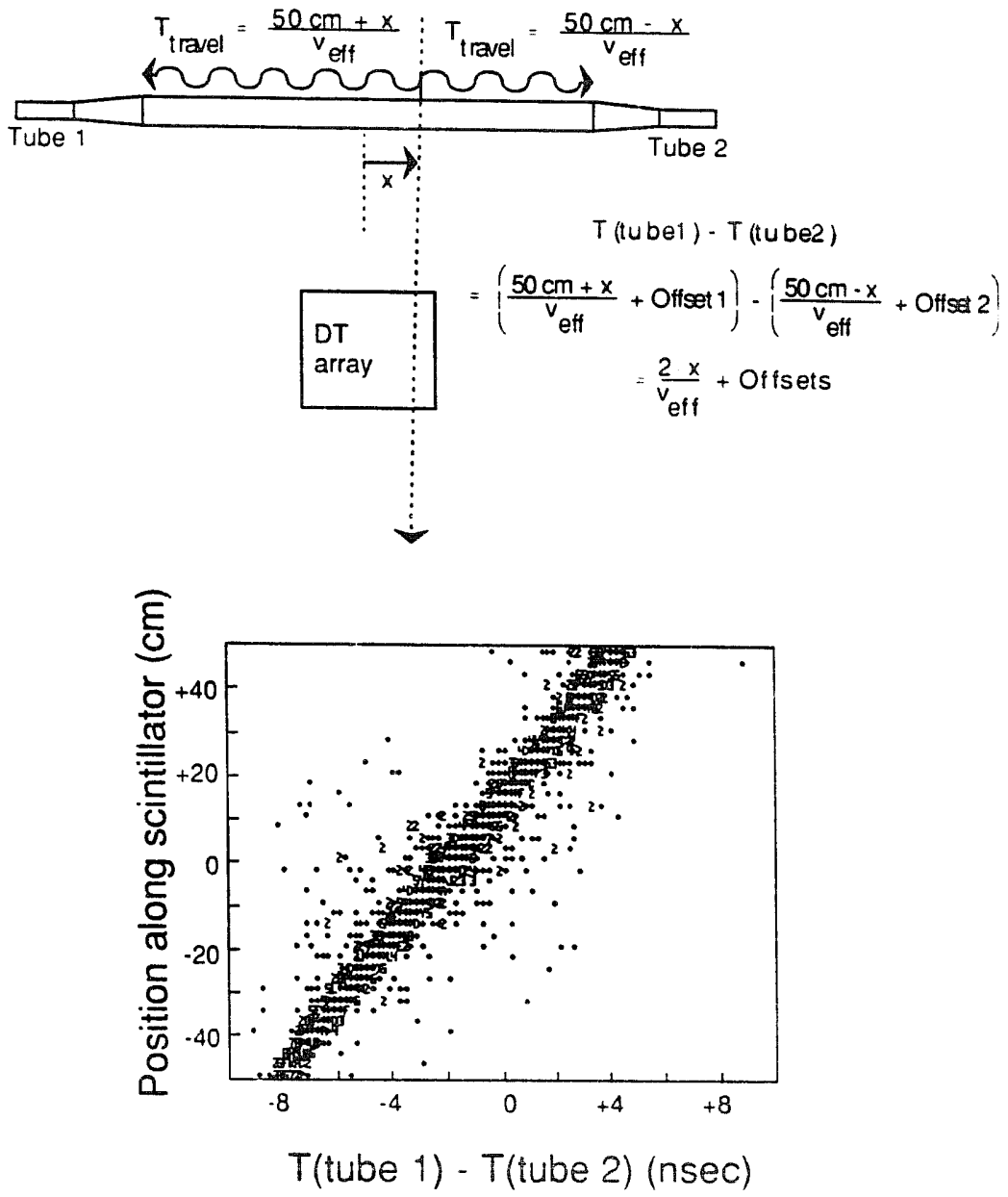

Fig. 31. Illustration showing the determination of particle position from the end-to-end timing difference registered by the two phototubes at opposite ends of a TOF scintillator. The scatter plot shown is made from flight proton data. The slope of the best-fit straight line to this scatter plot gives the effective speed of light propagation $t_{\mathrm{e} t}$ for the scintillator.

negative pions/muons are clearly identified, but no antiprotons are present. Had the goodness-of-fit cut on $\chi_{R}$ not been applied, hard scatterings would have populated the antiproton mass region yielding a spurious antiproton signal at the level of $\sim$ few $\times 10^{-4}$.

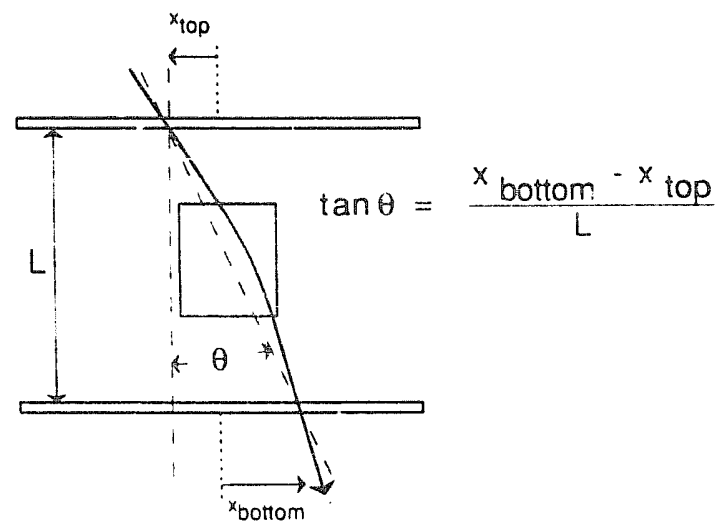

Fig. 32. Definition of the irack slope parameter $\tan \theta$.

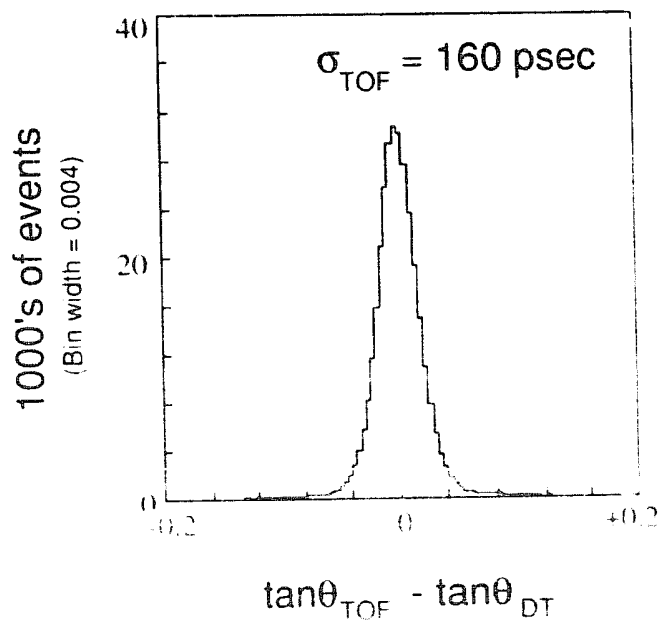

Fig. 33. Histogram of tangent slope difference for tracking and TOF systems used to infer $\sigma_{\text {TOF }}=160 \mathrm{ps}$ for flight data via the relation $\sigma_{\text {Tof }}=\left(L / v_{\text {eff }}\right) \sigma_{\Delta \tan \theta}$, where $L=150 \mathrm{~cm}$ is the separation between TOF counters, $v_{\text {eff }}$ is the effective propagation speed of light in the scintillator and $\theta$ in the track slope angle defined in fig. 32 . 


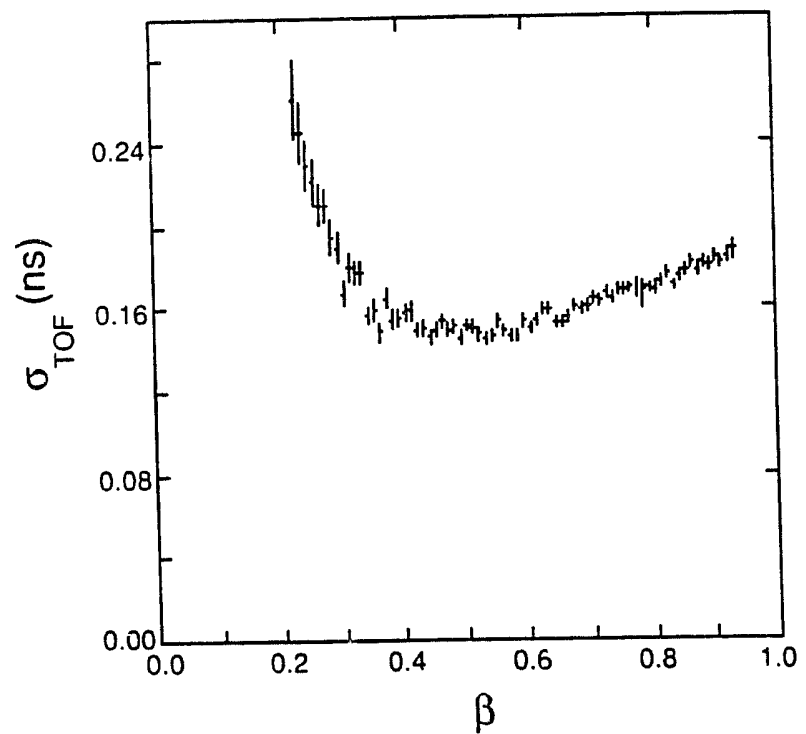

Fig. 34. TOF resolution as a function of $\beta$ for flight proton data.

As a final check, upward moving or "albedo" protons were selected (by virtue of their negative time of flight) for study to simulate antiproions, since an upward moving proton will exhibit exactly the same trajectory as a downward moving antiproton of the same rigidity. The resulting mass histogram is shown in fig. 38. Even though the momentum spectrum for albedo protons peaks at a much lower value than for down-

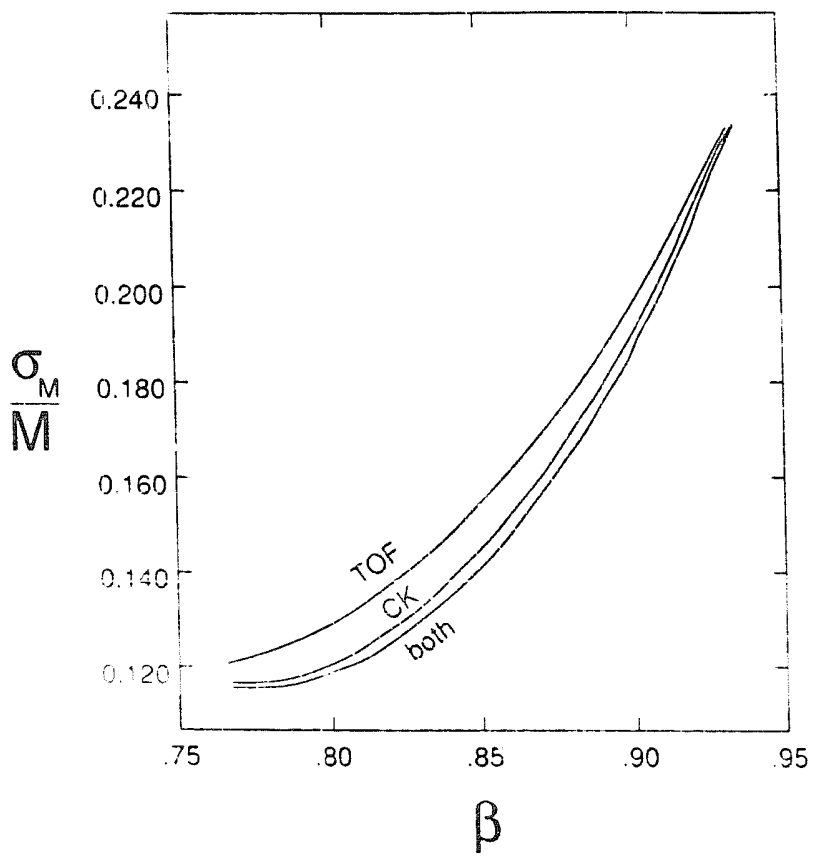

Fig. 35. Fractional mass resolution obtained for the velocity interval $0.8<\beta<0.93$ as a function of $\beta$ for velocity measured only with TOF, only with $C K$, and a variance-weighted average of the two (both)

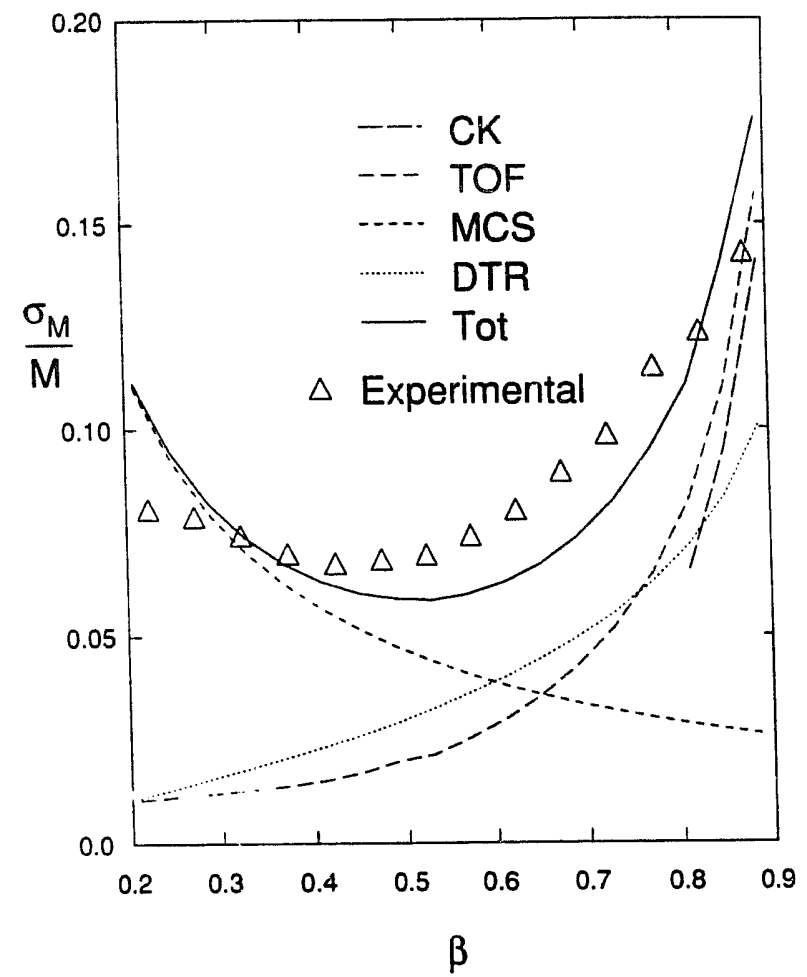

Fig. 36. Fractional mass resolution as a function of $\beta$ for flight data. Triangles report actual spectrometer resolution, while curves report contributions from Cherenkov velocity measurement (CK), time of flight velocity measurement (TOF), multiple Coulomb scattering (MCS) and drift tube tracking resolution (DTR). The solid curve reports the total (Tot) expected from all these contributions.

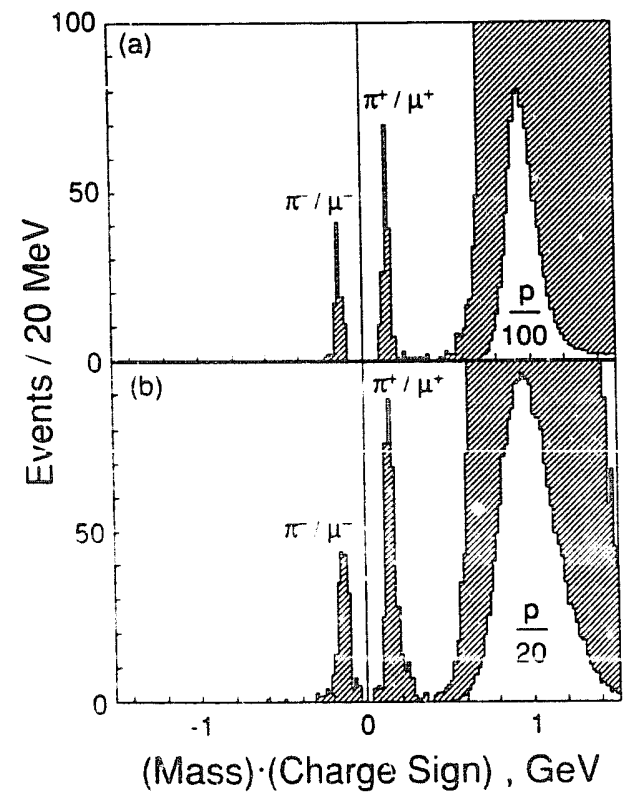

Fig. 37. Nas histograms for (a) $0.17<\beta<0.8$, and (b) $0.8<\beta$ $<0.93$. 


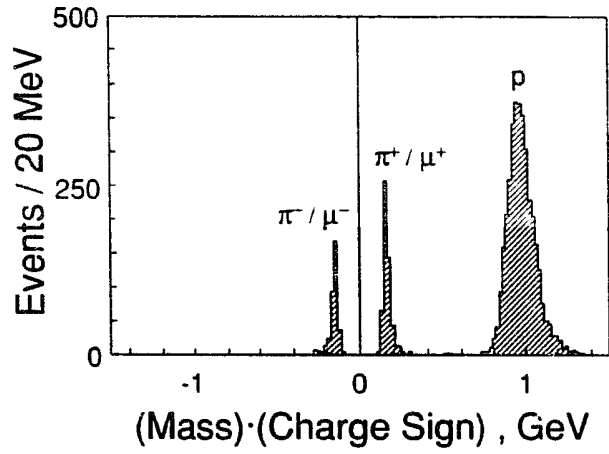

fig. 38. Mass histogram for albedo (upward moving) protons.

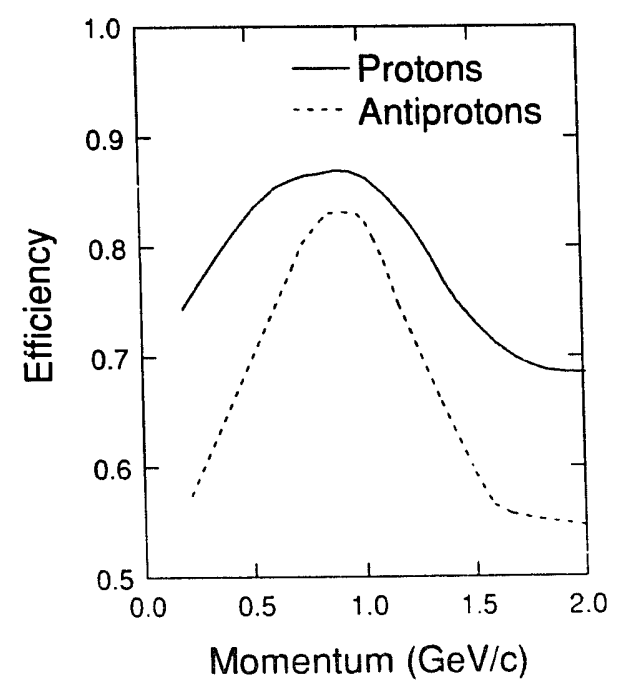

Fig. 39. Overall efficiency for the PBAR spectrometer for the detection of protons and antiprotons as a function of momentum, as determined from Monte Carlo studies.

ward moving protons, the mass distribution for the albedo sample peaks at almost the same values as the downward moving sample. Pions and muons are again tleanly separated from the protons. Since there are no charge asymmetric effects which would bias the scintillators against antiprotons [35], we conclude that the PBAR spectrometer did not discriminate against antiprotons beyond the corrections required for annihilation and scattering within the instrument. A detailed Monte Cárlo study [7] of the insirument has been performed, and provides the efficiency for antiprotons relative to protons when scattering and annihilation cross sections are taken into account for antiprotons propagating through the instrument. This efficiency as a function of mornentum is reported in fig. 39.

\section{Acknowledgements}

This experiment was made porsible by the efforts of personnel from the National Scientific Balloon Facility, and by financial assistance from Boston University, Indiana University, and the University of Michigan. We gratefully acknowledge assistance from J. Bartlett, A Buffington, R. Claxton, M. Gebhard, C. Foster, T. Karakashian, S. Lopez, S. Ma, D. O’Connell, H.S. Park, G. Pisello, J. Reynoldson, M. Solarz, C. Soulias, and G. Turner. This work was supported by National Science Foundation Grants PHY-8519440 (Boston University), PHY-8603225 and PHY-8702763 (University of California, Berkeley), U.S. Department of Energy Contract DE-AC02-76ER01112 (University of Michigan), NASA Contracts NGT-50014 (University of California, Berkeley), NAS1-17\%20 (University of Michigan), and NAGW1035 (Indiana University), and by Calspace grants CS78-87 and CS08-86 (University of California, Berkeley).

\section{References}

[1] A. Buffington, S.M. Schindler and C.R. Pennypacker, Astrophys. J. 248 (1981) 1179.

[2] J. Silk and M. S. ednicki, Phys. Rev. Lett. 53 (1984) 624.

[3] F.W. Stecker, S. Rudaz and T.F. Walsh, Phys. Rev. Lett. 55 (1985) 2622.

[4] J.S. Hagelin and G.L. Kane, Nucl. Phys. B263 (1986) 399.

[5] S. Rudaz and F.W. Stecker, Astrophys. J. 325 (1988) 16.

[6] S.P. Ahlen, S. Barwick, J.J. Beatty, C. R. Bower, G. Gerbier. R.M. Heinz, D. Lowder, S. McKee, S. Mufson, J.A. Musser, P.B. Price, M.H. Salamon. G. Tarlé, A. Tomasch and B. Zhou, Phys. Rev. Lett. 61 (1988) 145.

[7] M.H. Salamon, S.P. Ahlen, S. Barwick. J.J. Beatty, C.R Bower, G. Gerbier, R.M. Heinz, D. Lowder, S. McKee, S Mufson, J.A. Musser, P.B. Price, G. Tarlé. A. Tomasch and B. Zhou. Astrophys. J. 349 (1990) 78.

[8] R.L. Glukstern, Nucl. Instr. and Meth. 24 (1963) 381.

[9] M.H. Salamon, S.P. Ahlen and G. Tarlé. Phys. Rev. A21 (1980) 1506.

[10] E. Iarocci, Nucl. Instr. and Meth. 217 (1983) 30.

[11] J.J. Beatty et al., Proc. 20th Int. Cosmic Ray Conf., vol. 2 (1987) p. 374.

[12] C.C. Lo and B. Leskovar. IEEE Trans. Nucl. Sci. NS-28 (i 1981$) 659$

[13] T. Mass..m, Nucl. Instr. and Meth. 141 (1977) 251

[14] Available from Hartel Enterprises, Pacioma, CA. USA.

[15] Available from Champion Packages. Columbus. GA. USA.

[16] J. Incandella, S.P. Ahlen, J.J. Beatty, A. Ciccio, M. Felcini. D. Ficenec, E. Hazen. D. Levin. A. Marin, J.L. Stone. L.R. Sulak and $W$. Worsitl, Nucl. instr. and Meth. A26y (1988) 237 .

[17] Manufactured by Cryomagnetics Inc.. Oak Kidge. TN USA.

[18] A.D. Tomasch. Ph.D. Thesis, Boston University (1988).

[19] Manufactured by Precision Paper Tube. Wheeling, IL. USA.

[20] Manufactured by Emerson and Cuming, a division of W.R. Grace.

[21] Manufactured by California Fine Wire Co

[22] Manufactured by Milmax Inc. Oyster Bay, NY. USA. 
[23] M.H. Salamon, P.B. Price, D.M. Lowder et al., Proc. 23th Int. Conf. on High Energy Physics, Berkeley, vol. II (1986) p. 1302.

[24] S.P. Ahlen, B.G. Cartwright and G. Tarlé, Nucl. Instr. and Meth. 1.43 (1977) 513.

[25] C.R. Bower, R.M. Heinz, J. Reynoldson et al., Nucl. Instr. and Meth. A252 (1986) 112.

[26] C.R. Bower, Ph.D. Thesis, Indiana University (1988).

[27] V.R. Weidner, Appl. Opt. 25 (1986) 1265.

[28] J.D. Sullivan, Nucl. Instr. and Meth. 95 (1971) 5.

[29] D.M. Lowder, Ph.D. Thesis, University of Ca'ifornia at Berkeley (1988).
[30] A. Piesert and F. Sauli, CERN Report 84-08, Experimental Physics Division.

[31] G. Baranko, J.P. Guillaud, H. Ogren, D. Rust, S. Ems, S. Gray, B. Martin and P. Smith, Nucl. Instr. and Meth. 169 (1980) 413.

[32] H. Wind, Nucl. Instr. and Meth. 115 (1974) 431.

[33] G. D'Agostini, J.P. Albanese, J.J. Aubert, C. Benchouck, E. Kajfasz, R. Nacasch and P. Payre, Nucl. Instr. and Meth. 219 (1984) 495.

[34] Review of Particle Properties, Phys. Lett. B170 (1986) 45.

[35] S.P. Ahlen, Rev. Mod. Phys. 52 (1980) 121. 